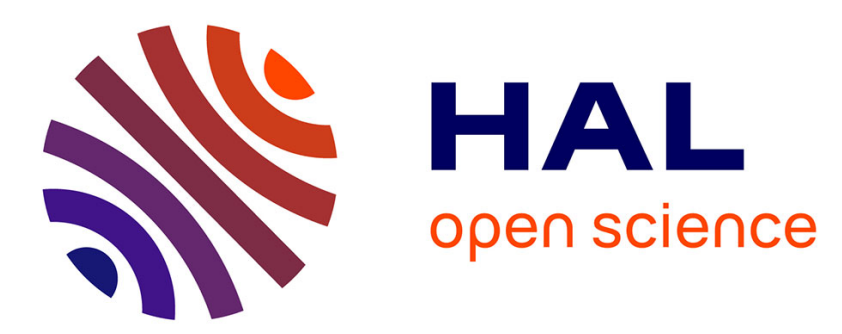

\title{
Non-linear vibrations of free-edge thin spherical shells: Experiments on a 1:1:2 internal resonance
}

Olivier Thomas, Cyril Touzé, Éric Luminais

\section{To cite this version:}

Olivier Thomas, Cyril Touzé, Éric Luminais. Non-linear vibrations of free-edge thin spherical shells: Experiments on a 1:1:2 internal resonance. Nonlinear Dynamics, 2007, 49 (1-2), pp.259-284. 10.1007/s11071-006-9132-y . hal-00838881

HAL Id: hal-00838881

https://hal-ensta-paris.archives-ouvertes.fr/hal-00838881

Submitted on 12 Jul 2016

HAL is a multi-disciplinary open access archive for the deposit and dissemination of scientific research documents, whether they are published or not. The documents may come from teaching and research institutions in France or abroad, or from public or private research centers.
L'archive ouverte pluridisciplinaire HAL, est destinée au dépôt et à la diffusion de documents scientifiques de niveau recherche, publiés ou non, émanant des établissements d'enseignement et de recherche français ou étrangers, des laboratoires publics ou privés. 


\title{
Non-linear vibrations of free-edge thin spherical shells: Experiments on a 1:1:2 internal resonance
}

\author{
O. Thomas · C. Touzé · É. Luminais
}

\begin{abstract}
This study is devoted to the experimental validation of a theoretical model of large amplitude vibrations of thin spherical shells described in a previous study by the same authors. A modal analysis of the structure is first detailed. Then, a specific mode coupling due to a 1:1:2 internal resonance between an axisymmetric mode and two companion asymmetric modes is especially addressed. The structure is forced with a simple-harmonic signal of frequency close to the natural frequency of the axisymmetric mode. The experimental setup, which allows precise measurements of the vibration amplitudes of the three involved modes, is presented. Experimental frequency response curves showing the amplitude of the modes as functions of the driving frequency are compared to the theoretical ones. A good qualitative agreement is obtained with the predictions given by the model. Some quantitative discrepancies are observed and discussed, and improvements of the model are proposed.
\end{abstract}

Keywords Shallow spherical shells · Geometrical nonlinearities · Internal resonance $\cdot$ Non-linear vibrations $\cdot$ Experiments

O. Thomas $(\bowtie)$

Structural Mechanics and Coupled Systems Laboratory, CNAM, 2 rue Conté, 75003 Paris, France

e-mail: olivier.thomas@cnam.fr

C. Touzé

ENSTA-UME, Chemin de la Hunière, 91761 Palaiseau

Cedex, France

\section{Introduction}

When a thin shell is subjected to large amplitude vibrations, of the order of magnitude of the thickness, geometrical nonlinearities cannot be neglected and give rise to numerous nonlinear phenomena [1]. Among these, nonlinear interactions between modes can result in transfers of energy among them. In a structural dynamics context, these phenomena are important because an excitation of the structure in a specific frequency band can give rise to large amplitude oscillations at other frequencies. Moreover, a spatial redistribution of energy can be observed from one mode shape to another one, and parts of the structure not excited at the primary resonance can receive energy through the coupling and oscillate at large amplitude. Finally, such a modal interaction is a usual way for a continuous structure to transit to more complex dynamics and chaos $[2,3]$. This article presents a series of measurements of a special nonlinear coupling between three modes in one-to-one-to-two (1:1:2) internal resonance, in the case of forced vibrations of a spherical shell.

In the abundant literature devoted to nonlinear vibrations of shells (see for example the reviews of Qatu [4], Amabili and Païdoussis [5] and Alhazza and Alhazza [6]), only a few studies propose experimental investigations, as compared to the number of theoretical works available. Moreover, the case of shallow spherical shells is generally reduced to axisymmetric vibrations, whereas asymmetric modes are of prime importance to understand the global dynamics exhibited: 
they are numerous as compared to the axisymmetric ones, and since nonlinear couplings between any modes are possible [7], an axisymmetric excitation of the structure can lead to asymmetric vibrations. Experiments on axisymmetric as well as asymmetric vibrations of a clamped spherical cap are proposed in [8], but this study is restricted to linear vibrations. Non-linear experimental studies on shells generally focus on onemode vibrations and on the associated hardening or softening behavior. Amabili et al. [9, 10] experimentally investigated nonlinear vibrations of cylindrical shells, coupled with fluid or not, and reported other experimental works. On spherical shells, experimental results on snap-through behavior are exposed in [11] and a few qualitative experiments on the special case of a one-to-two (1:2) internal resonance between two axisymmetric modes are reported in [12]. To the knowledge of the authors, no experiments on multi-mode asymmetric nonlinear response of spherical shells have been proposed yet.

The aim of the present study is precisely to investigate experimentally energy transfers via a nonlinear mode coupling between axisymmetric and asymmetric modes. A complete theoretical model, including asymmetric vibrations, has been derived in [7], where the particular case of a 1:1:2 internal resonance has been theoretically investigated. The 1:1:2 internal resonance involves in our study a modal interaction between two companion asymmetric modes, which have nearly equal natural frequencies $f_{1} \simeq f_{2}$ (thus being in oneto-one (1:1) internal resonance) and one axisymmetric mode, whose natural frequency $f_{3}$ is close to twice the ones of the asymmetric companion modes (all three being in 1:1:2 internal resonance, $f_{1} \simeq f_{2} \simeq f_{3} / 2$ ). The measurements presented here have been obtained with a spherical cap with a free edge, driven by a simple-harmonic force at its center, in the vicinity of the resonance of the axisymmetric mode. A transfer of energy toward the asymmetric companion modes is observed, those modes oscillating at half the driving frequency, thus creating a subharmonic in the shell response. A 1:1 internal resonance between two asymmetric modes has already been studied by the authors in the case of a circular plate, theoretically in [13] and experimentally in [14]. The experimental setup used here shares some common feature with the one used in [14].

The outline of the paper is now exposed. Some experimental details used throughout the paper are firstly exposed in Section 2. Then, Section 3 provides a modal analysis that allows precise measurements of all the linear parameters of the shell under study. Those parameters are compared to the ones obtained theoretically in [7]. Section 4 briefly recalls the vibratory behavior of the shell in forced 1:1:2 internal resonance as predicted by the theory exposed in [7]. In Section 5, the experimental setup that allows the measurements of the nonlinear behavior of the shell is detailed. The experimental results are exposed in Section 6 and compared to theory in Section 7. An excellent qualitative agreement is obtained between theory and experiments. However, some quantitative discrepancies are observed, and some improvements of the theoretical model are finally suggested.

\section{Experimental details}

\subsection{Geometry of the shell}

In order to draw out precise comparisons between the theoretical results and the experiments, a shell with a nearly circular profile has been built. Its geometrical characteristics are as follows: its outer diameter is $2 a=600 \mathrm{~mm}$, thickness is $h=1 \mathrm{~mm}$, center height is $H=30 \mathrm{~mm}$, and radius of curvature is approximately $R=1515 \mathrm{~mm}$ (Fig. 1). It is made of brass of Young's modulus $E$, Poisson's ratio $v$, and density $\rho$, supposed to be homogeneous and isotropic. Three small holes (of diameter $2 \mathrm{~mm}$ ) at the rim allow us to hang up the shell with nylon threads.

A precise measurement of the shell profile has been performed with a dial comparator. The results are presented on Fig. 2. Two circular profile are also drawn, one with a radius of curvature $R=1515 \mathrm{~mm}$, the one announced by the maker, and one that has been estimated by fitting a circle to the experimental points ( $R=1557 \mathrm{~mm}$ ). One can observe that the profile of the shell is close to a circle but that its curvature is not uniform.

\subsection{Boundary conditions}

In order to simulate free-edge boundary conditions, the shell is held with two nylon threads, fixed by means of small holes at the rim (Fig. 3). 
Fig. 1 Geometry of the shell

Fig. 2 Profile of the shell. (० ०) Measured profile. (- -) Spherical profile with $R=1.515$ m. (- - -)

Spherical profile with $R=1.557 \mathrm{~m}$
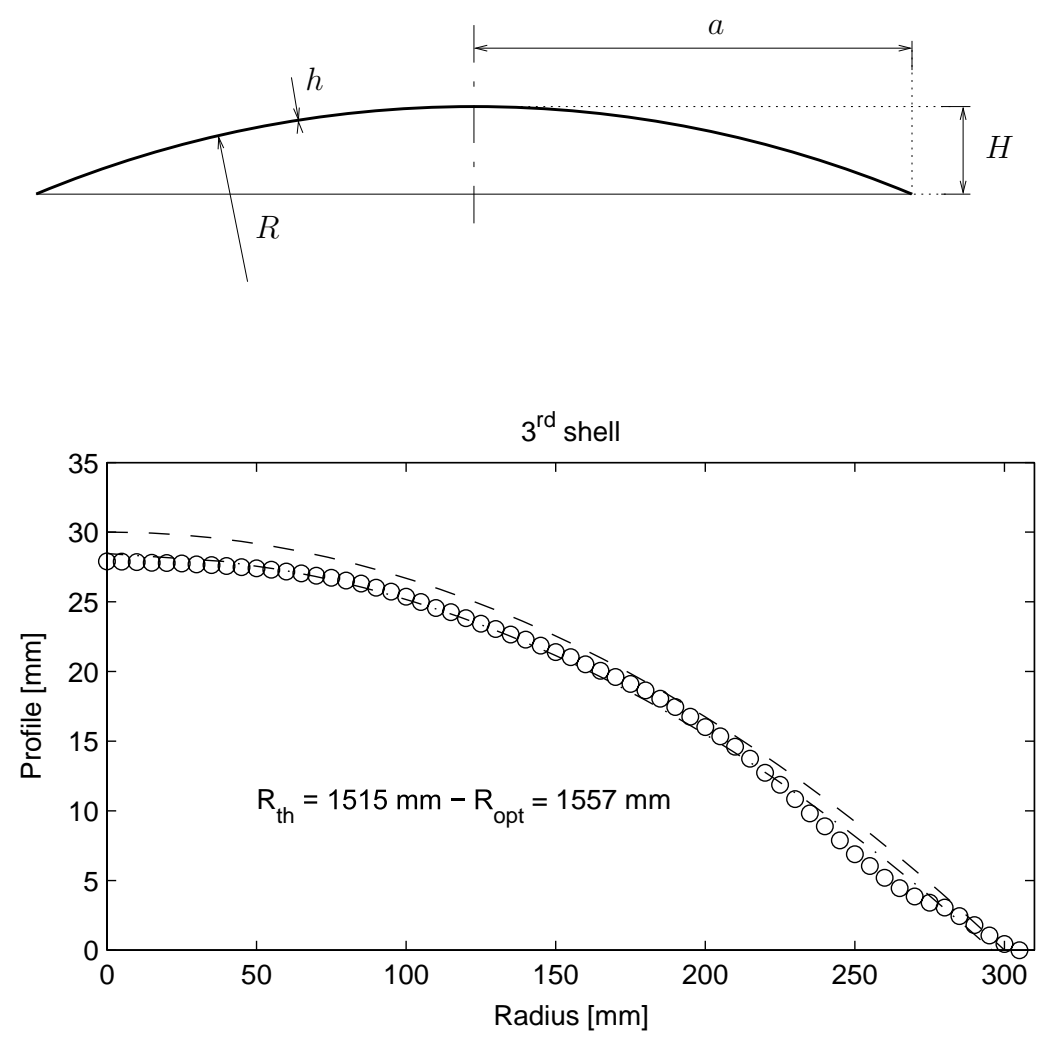

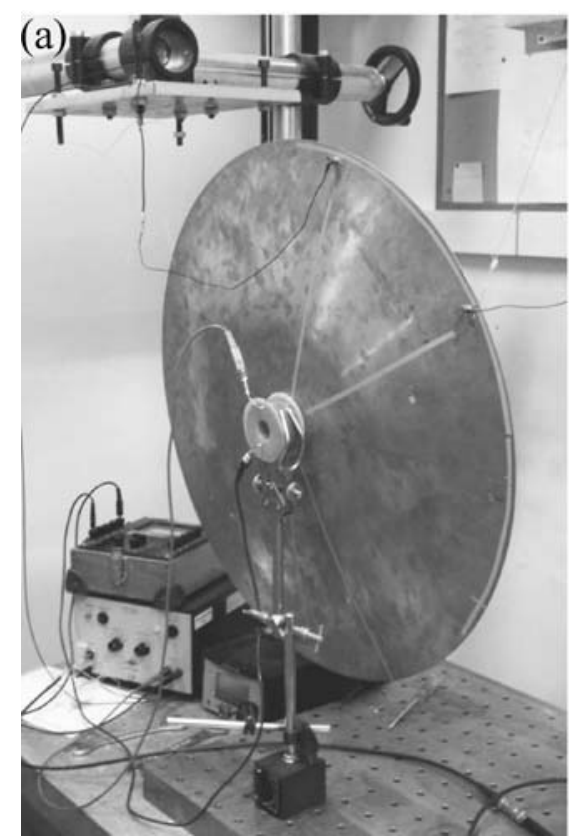

(b)

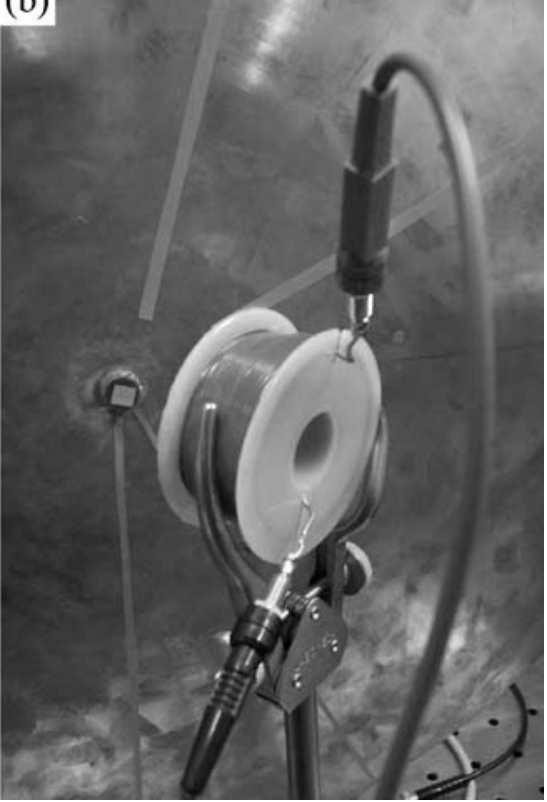

Fig. 3 Photographs of the experimental setup. (a) The spherical cap with excitation device and accelerometers. (b) Detail of the coil-magnet exciter 
Table 1 Devices used during the modal analyzes

Fig. 4 Electromagnetic exciter. The magnet is radially centered in the coil cavity. Dimensions are in millimeter

\begin{tabular}{lll}
\hline Noise synthesizer & Brüel \& Kjær & PULSE \\
Power amplifier & QSC & PLX 3402 \\
Scanning laser vibrometer & Polytec & OFV 056/OFV 3001 S \\
Deflection shape estimation & Polytec & PSV 300 \\
\hline
\end{tabular}

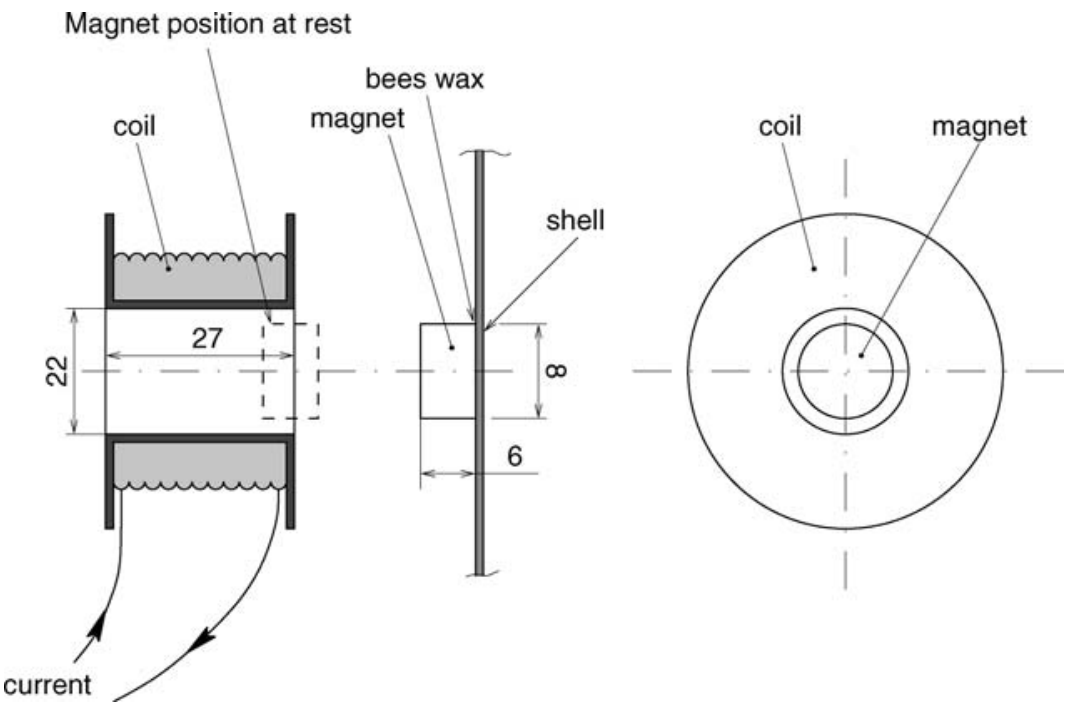

\subsection{Excitation device}

The shell is excited by means of a magnet, glued with beeswax and driven by a coil (Figs. 3(b) and 4). The coil is fed through a power amplifier by either a random noise for the modal analyzes (Section 3) or by a sine signal for the nonlinear experiments (Section 6). This excitation device has already been used in [14], where the interested reader can find a precise description of the calibration procedure that allows to evaluate the force acting on the magnet as a function of the current intensity in the coil. It has been found that the force is proportional to the intensity, under the condition that the magnet has a constant position with respect to the coil. Proportionality coefficient $K$ depends on the position of the magnet with respect to the coil. As the magnet follows the shell oscillations during experiments, the force is actually not purely proportional to the intensity. In particular, when the current is simple-harmonic, harmonic distortion of the force signal occurs. However, the lowest harmonic distortion is obtained by adjusting precisely the position at rest of the magnet so that the symmetry plane of the magnet coincides with the side plane of the coil. The harmonic distortion is in this case of the order of $1 \%$ for a magnet displacement amplitude of the order of $2.5 \mathrm{~mm}$ [14], which is much greater than the maximum amplitudes encountered during the nonlinear experiments (see Section 6: the amplitude of the center of the shell is less than $0.5 \mathrm{~mm}$ in Fig. 16). The magnet is also radially centered with respect to the coil. No force transducer is used in the experiments: the force acting on the magnet and thus on the shell is estimated by measuring the intensity of the current in the coil and by multiplying it with coefficient $K$.

\section{Modal analysis of the shell}

In this section, an experimental modal analysis of the shell is presented. The parameters related to the linear vibrations of the shell are estimated and compared to the theory exposed in [7]. Those parameters will be used in Section 7 to compare the theoretical model of nonlinear vibrations to the experimental results.

\subsection{Experiments}

The devices used are listed in Table 1. The shell is driven by a random noise signal through the coil/magnet exciter. The driving amplitude is chosen weak enough to ensure linear vibrations of the shell. Two locations for the magnet (of mass $6 \mathrm{~g}$ ) have been 


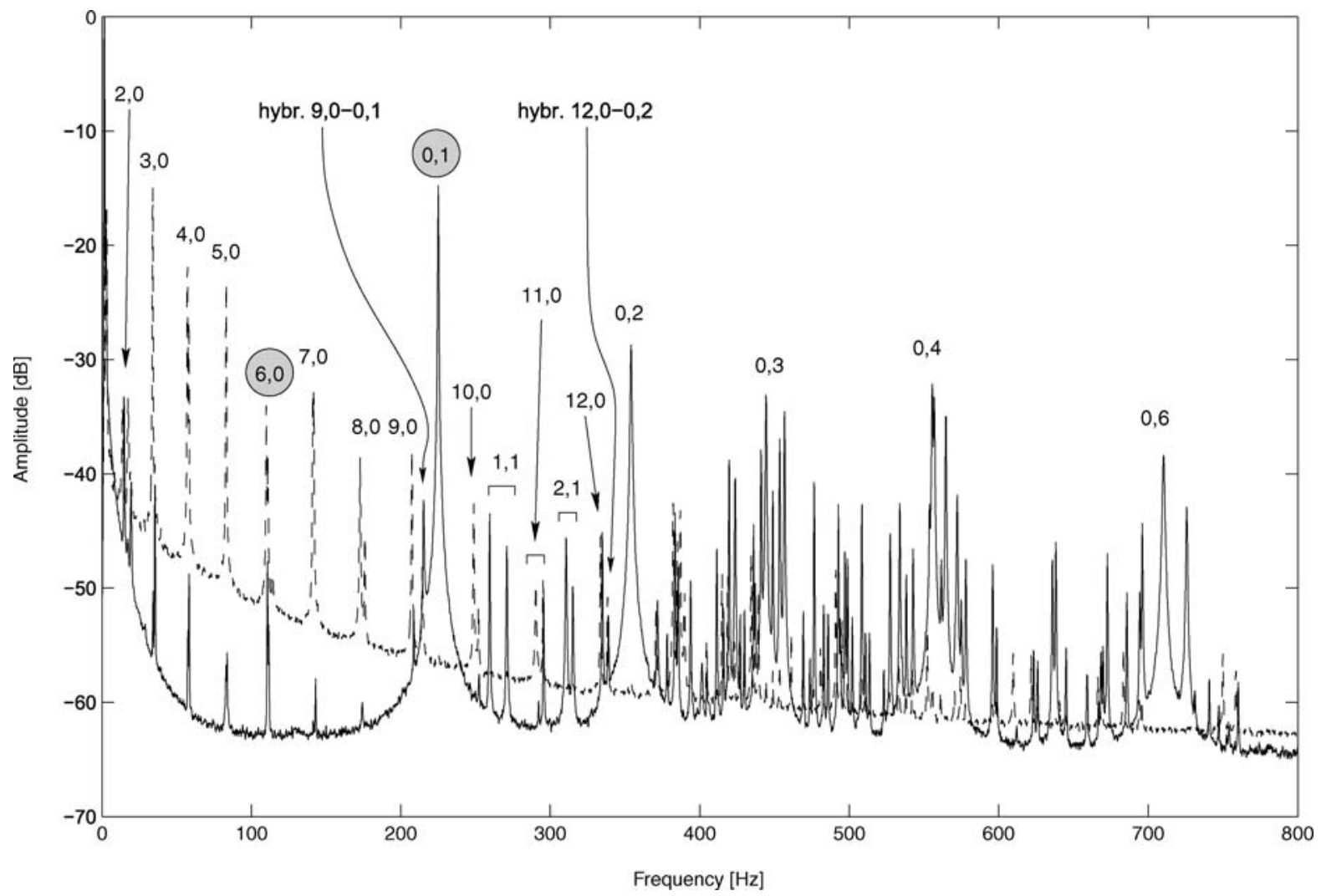

Fig. 5 Frequency response of the shell. (-) Center excitation. (--) Rim excitation

chosen: the first one at the center of the shell to drive mainly the axisymmetric modes, and the second one at the rim, $10 \mathrm{~mm}$ from the edge of the shell, to drive the other modes.

The velocity response of the shell is measured with a laser vibrometer, at any point of a $40 \times 40$ point square grid. At each point, the power spectral density (PSD) of the measured signal is estimated. Then, three types of data are obtained:

- A mean PSD, obtained by averaging all the PSDs obtained for all the grid points. Figure 5 shows the PSDs for both excitation locations.

- The values of the natural frequencies of the structure, obtained by the values of the peak frequencies of the curves of Fig. 5.

- Various operational deflection shapes, obtained at the peak frequencies of the curves of Fig. 5.

One can notice that the measured natural frequencies and mode shapes correspond to the spherical shell with the added mass brought by the magnet.

\subsection{Comparison with theory}

By observing the deflection shapes and comparing them to the theoretical ones obtained in [7], it is possible to identify most of the resonant frequencies. As in the theory, three mode families are obtained:

- the purely asymmetric modes display only nodal diameters (no nodal circles),

- the axisymmetric modes display only nodal circles (no nodal diameters),

- the mixed modes are the others.

The number $(k, n)$ of nodal diameters $(k)$ and circles $(n)$ of the modal shape associated with each natural frequency is indicated in Fig. 5, the corresponding values of the natural frequencies are listed in Table 2, and some measured deflection shapes along with the corresponding profiles are shown in Fig. 6. We can observe that as the geometry of the shell is nearly axisymmetric, all modes except the axisymmetric ones appear by pair in the spectrum, the two members of a 


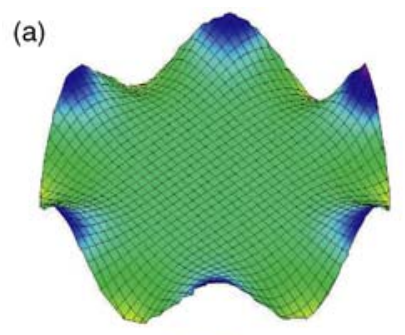

(b)

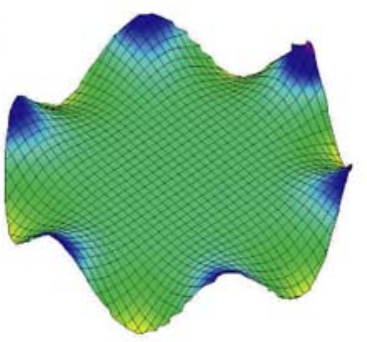

(d)
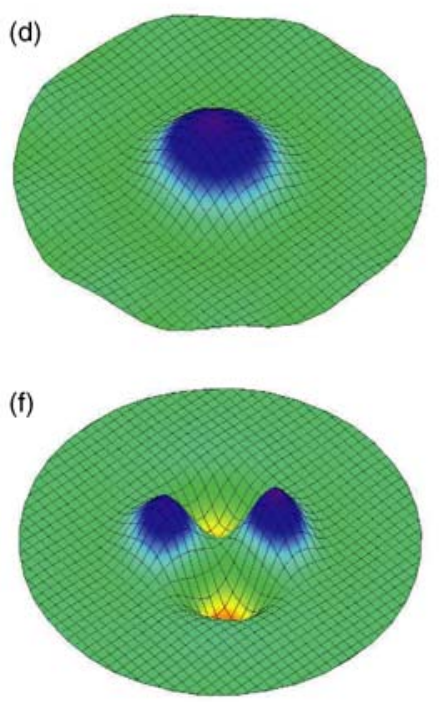

(g)

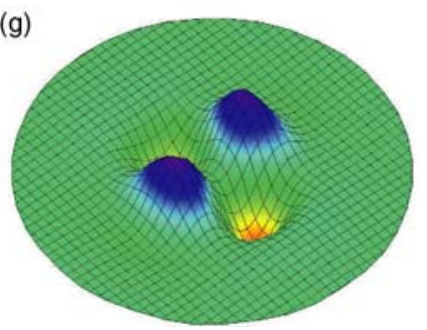

Fig. 6 Three-dimensional view of the experimental deflection shape. (a), (b) Both companion asymmetric modes $(6,0)$. (d) Axisymmetric mode $(0,1)$. (f), (g) Both companion mixed mode

pair being denoted as companion modes. If the structure had a perfectly axisymmetric geometry, those companion modes would have exactly the same natural frequencies. For this shell, the purely asymmetric modes $(k, 0)$ show natural frequencies very close to each
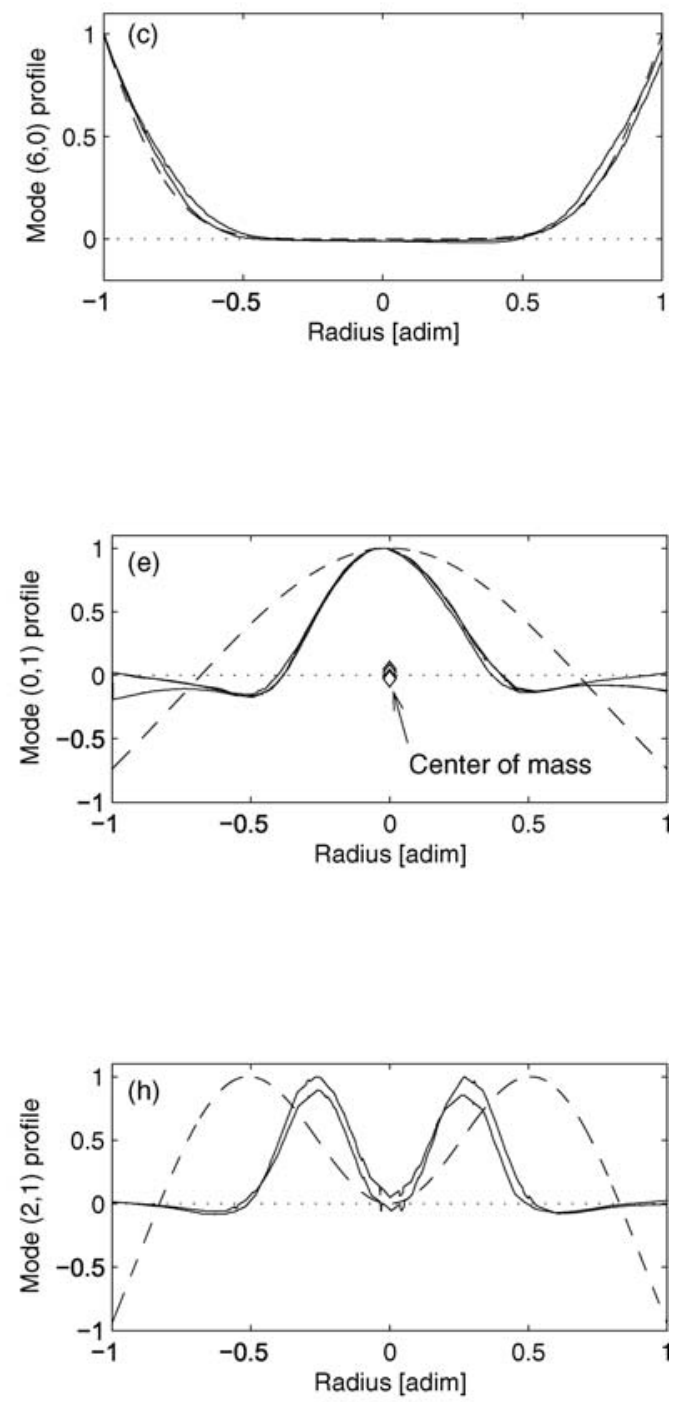

$(2,1)$. (c), (e), (h) Corresponding profiles for two diameters. (-) Experimental. (--) Theoretical

other, whereas some mixed companion modes can have spaced natural frequencies. Moreover, in the theory, the mode shapes of two companion modes have the same geometry, the only difference lying in their angular position: the nodal diameters of one companion mode are 
Table 2 Values of experimental and theoretical natural frequencies of the shell

\begin{tabular}{llll}
\hline $\begin{array}{l}\text { Mode } \\
\text { number }\end{array}$ & \multicolumn{2}{c}{$\begin{array}{c}\text { Experimental } \\
\text { frequency }(\mathrm{Hz})\end{array}$} & $\begin{array}{l}\text { Theoretical } \\
\text { frequency }(\mathrm{Hz})\end{array}$ \\
\hline$(2,0)$ & 13.75 & 17.5 & 11.02 \\
$(3,0)$ & 34 & 35.5 & 26.37 \\
$(4,0)$ & 57.25 & 58.25 & 46.90 \\
$(5,0)$ & 83 & 83.75 & 72.17 \\
$(6,0)$ & 110 & 111 & 101.77 \\
$(7,0)$ & 141 & 141.8 & 135.45 \\
$(8,0)$ & 172.75 & 176 & 173.01 \\
$(9,0)$ & 207 & 214 & 214.39 \\
$(10,0)$ & 248.75 & 252 & 259.57 \\
$(11,0)$ & 290.25 & 295 & 308.60 \\
$(12,0)$ & 333.75 & 338.25 & 361.53 \\
$(0,1)$ & \multicolumn{2}{c}{225} & 386.03 \\
$(0,2)$ & \multicolumn{2}{c}{354} & 393.11 \\
$(0,3)$ & \multicolumn{2}{c}{444.25} & 423.17 \\
$(0,4)$ & \multicolumn{2}{c}{555.5} & 495.65 \\
\hline
\end{tabular}

located at the maxima of the other. This property has been qualitatively verified in the experiments, as it can be observed in Fig. 6 .

Figures 5 and 7(a), and Table 2 show qualitatively that the asymmetric mode $(k, 0)$ natural frequencies are arranged in the spectrum in an analogous manner compared to the corresponding theoretical ones obtained in [7]. Moreover, it has been verified that the experimental radial dependence of the asymmetric mode shapes (their profiles) fit the corresponding theoretical ones very well. For example, the comparison between experimental and theoretical profile for mode $(6,0)$ is shown in Fig. 6(c). In contrast, Fig. 7(b) and Table 2 show that the experimental natural frequencies of the axisymmetric modes, as well as the ones of the mixed modes, poorly fit their corresponding theoretical values. An example is $f_{01}$, the frequency of the first axisymmetric mode, whose experimental value is $160 \mathrm{~Hz}$ lower than in the theory. It has also been observed that the deformed part of the deflection shapes of those modes are all located in the half central part of the structure and that the corresponding profiles poorly fit the corresponding theoretical ones, as it is shown in Figs. 6(e) and $6(\mathrm{~h})$ for mode $(0,1)$ and mode $(2,1)$. This fact can probably be explained by the geometry of the shell profile, which is not perfectly circular (Fig. 2). A conclusion is that the slight imperfections of the geometrical profile of the shell have a major influence on the modes with at least one nodal circle (the axisymmetric modes as well as the mixed modes), whereas they do not significantly influence the purely asymmetric modes.

For any structure with perfectly free boundary conditions and no rigid body motions, the center of mass stays motionless during the oscillations. It is a consequence of the second Newton's law applied to the structure, free of external forces. Thus, if the boundary conditions are perfectly free, the center of mass of any deformed shapes is located on the plane of the vibration nodes. The positions of the center of mass of each experimental profiles of mode $(0,1)$ have been computed and are shown in Fig. 6(e). The details of the computation can be found in Appendix 8. Figure 6(e) shows that the center of mass is located very close to the zero axis. One can conclude that our experimental setup realizes boundary conditions that are very close to a free edge and that, consequently, the associated imperfections probably play a negligible role in the discrepancies between theory and experiments.

The relation between the natural frequencies $f_{k n}$ and the dimensionless theoretical angular frequencies $\bar{\omega}_{k n}$ is:

$$
f_{k n}=\underbrace{\frac{h}{2 \pi a^{2}} \sqrt{\frac{E}{12 \rho\left(1-v^{2}\right)}}}_{f^{*}} \bar{\omega}_{k n} .
$$

A value of coefficient $f^{*}=1.985 \mathrm{~Hz}$ has been estimated by the slope of the least-square straight line of Fig. 7(a) that approximates the values of the asymmetric natural frequencies. By measuring the mass of the structure, the value of the density of the material has been evaluated to $\rho=8230 \mathrm{~kg} \mathrm{~m}^{-3}$. Using Equation (1) with $v=0.33$, one obtains $E=111 \mathrm{GPa}$ for the Young's modulus of the material.

\section{Theoretical behavior of the shell in 1:1:2 internal resonance}

This section briefly recalls the main results obtained in [7], allowing theoretical prediction of the vibratory behavior of the shell.

\subsection{Theoretical model}

The dimensionless equations of motion for the shell (dimensionless quantities are denoted by over-bars), in 
Fig. 7 (a) Dimensionless asymmetric theoretical angular frequencies $\bar{\omega}_{k 0}$ of the shell (from [7]) as a function of the corresponding experimental natural frequencies $f_{k 0}^{\exp }$.

The slope of the fitting line is $f^{*}=1.985 \mathrm{~Hz}$. (b)

Difference between experimental and theoretical frequencies, $f_{k n}^{\exp }-f_{k n}^{\text {the }}$, as a function of $f_{k n}^{\exp }$. (c)

Difference between experimental and theoretical frequencies,

$\left(f_{k n}^{\text {exp }}-f_{k n}^{\text {the }}\right) / f_{k n}^{\text {the }}$, as a function of $f_{k n}^{\text {exp }}$, in percent. (०) Asymmetric modes, from $(2,0)$ to $(12,0) .(\triangle)$ Axisymmetric modes, from $(0,1)$ to $(0,4)$. $(\star)$ Mixed modes $(1,1)$ and $(2,1)$
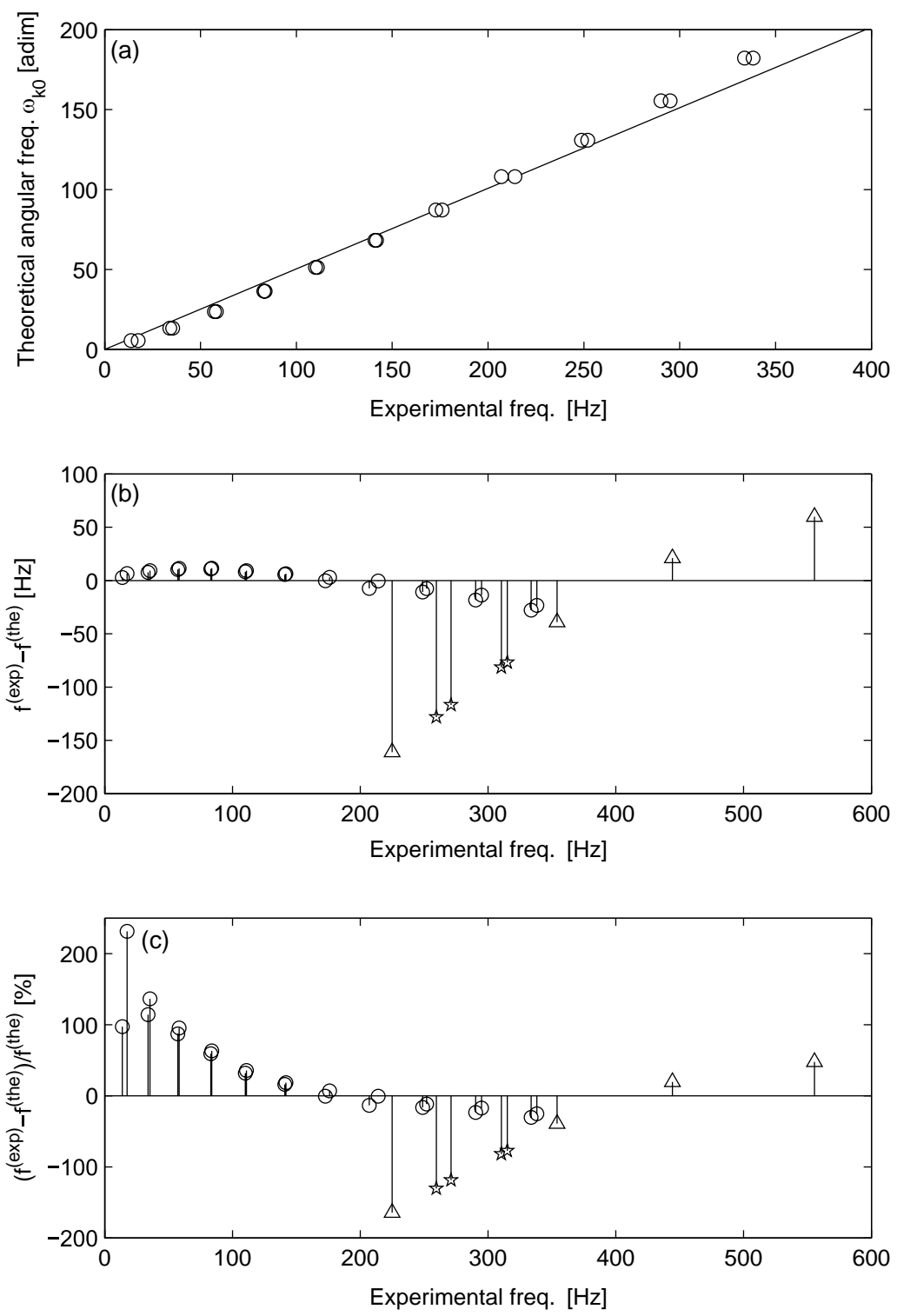

terms of transverse displacement $\bar{w}$ along the normal to the mid-surface and airy stress function $\bar{F}$, write for all time $\bar{t}$ :

$$
\begin{aligned}
\Delta \Delta \bar{w}+\varepsilon_{q} \Delta \bar{F}+\ddot{\bar{w}}= & \varepsilon_{c} L(\bar{w}, \bar{F}) \\
& +\varepsilon_{q}[-2 \mu \dot{\bar{w}}+\bar{p}], \\
\Delta \Delta \bar{F}-\frac{a^{4}}{R h^{3}} \Delta \bar{w}= & -\frac{1}{2} L(\bar{w}, \bar{w}),
\end{aligned}
$$

where $\Delta$ stands for the Laplacian operator, $\ddot{\bar{w}}$ denotes the second time derivative of $\bar{w}$, and $\mu$ is a viscous damping coefficient. These equations correspond to a generalization to the case of a curved geometry of von Kármán's model for large-deflection vibrations of plates [15]. For any detail on the assumptions, the freeedge boundary conditions and other references on the subject, the interested reader can refer to [7]. $\varepsilon_{q}$ and $\varepsilon_{c}$ are two "small" parameters that scale respectively the quadratic and cubic terms in the equations of motion. They fulfill the following relationship [7]:

$\varepsilon_{c}=\frac{\varepsilon_{q}^{2}}{\chi}$ 
where $\chi=12\left(1-v^{2}\right) a^{4} / R^{2} h^{2}$ is a geometrical parameter.

The local dynamic partial differential Equations (2a) and ( $2 b)$ are expanded onto the linear mode basis. It is then found that the corresponding modal coordinates are solutions of an infinity of second order ordinary differential equations coupled by quadratic and cubic nonlinear terms [7]. A particular 1:1:2 internal resonance between two companion purely asymmetric modes (denoted in the following by mode 1 and mode 2) of dimensionless natural frequencies $\bar{\omega}_{1}$ and $\bar{\omega}_{2}$ and an axisymmetric mode (mode 3 ) of dimensionless natural frequency $\bar{\omega}_{3}$ is studied. This internal resonance occurs if:

$\bar{\omega}_{3} \simeq 2 \bar{\omega}_{1} \simeq 2 \bar{\omega}_{2}$

Considering Equation (3), a first-order solution to this particular 1:1:2 internal resonance is obtained by retaining only the quadratic nonlinear terms and the three modal coordinates corresponding to the three modes involved in the internal resonance. This drastic truncation allows derivation of the simplest model; in fact, the normal form of the 1:1:2 internal resonance, which describes the first bifurcations of the system. Its validity is also assessed by the fact that this simple truncation captures all the qualitative features exhibited by the experiments, as it will be shown in Section 6. The dimensionless displacement of the shell in steady state is then [7]:

$$
\begin{aligned}
\bar{w}(\bar{r}, \theta, \bar{t})= & \Phi_{1}(\bar{r}, \theta) q_{1}(\bar{t}) \\
& +\Phi_{2}(\bar{r}, \theta) q_{2}(\bar{t})+\Phi_{3}(\bar{r}) q_{3}(\bar{t}),
\end{aligned}
$$

where $(\bar{r}, \theta)$ denote the usual dimensionless polar coordinates and $\left(\Phi_{i}, q_{i}\right), i=1,2,3$ denote the mode shape and the modal coordinate associated with mode $i$. When the structure is driven at its center by a harmonic forcing of angular frequency $\bar{\Omega}$, the modal coordinates $q_{i}$ verify the following dynamical equations:

$$
\begin{aligned}
& \ddot{q}_{1}+\bar{\omega}_{1}^{2} q_{1}=\varepsilon\left[\alpha_{1} q_{1} q_{3}-2 \mu_{1} \dot{q}_{1}\right] \\
& \ddot{q}_{2}+\bar{\omega}_{1}^{2} q_{2}=\varepsilon\left[\alpha_{2} q_{2} q_{3}-2 \mu_{2} \dot{q}_{2}\right] \\
& \ddot{q}_{3}+\bar{\omega}_{1}^{2} q_{3}=\varepsilon\left[\alpha_{3} q_{1}^{2}+\alpha_{4} q_{2}^{2}-2 \mu_{3} \dot{q}_{3}+Q \cos \bar{\Omega} \bar{t}\right],
\end{aligned}
$$

where $\varepsilon=\varepsilon_{q},\left\{\alpha_{i}\right\}_{i=1, \ldots, 4}$ are the coefficients related to the mode shapes of the shell and $Q$ is related to the forcing amplitude. $\left\{\mu_{i}\right\}_{i=1,2,3}$ are the modal damping coefficients of the three involved modes, related to damping factors $\left\{\xi_{i}\right\}_{i=1,2,3}$ by, for all $i=1,2,3$ :

$\mu_{i}=\frac{\xi_{i} \bar{\omega}_{i}}{\varepsilon}$.

Their values can be estimated by experimental modal analyzes and will be presented in Section 7. One can notice that no forcing term appears in Equations (6a) and (6b) because modes 1 and 2 are asymmetric and thus their modal shapes have nodes at the center (Fig. 6). Moreover, thanks to the few parameters involved in Equations (6a) and (6b), their solutions can easily be fitted to experimental nonlinear responses in order to estimate the nonlinear coefficients $\left\{\alpha_{i}\right\}_{i=1, \ldots, 4}$, so as to compare them to the theoretical ones obtained in [7]. This will be presented in Section 7.

When the structure is driven in the vicinity of the resonance of mode $3\left(\bar{\Omega} \simeq \bar{\omega}_{3}\right)$, a first-order solution to the set of equations (6a)-(6c), obtained by the method of multiple scales in [7], is:

$$
\begin{aligned}
& q_{1}(\bar{t})=\bar{a}_{1} \cos \left(\frac{\bar{\Omega}}{2} \bar{t}-\frac{\gamma_{1}+\gamma_{3}}{2}\right), \\
& q_{2}(\bar{t})=\bar{a}_{2} \cos \left(\frac{\bar{\Omega}}{2} \bar{t}-\frac{\gamma_{2}+\gamma_{3}}{2}\right), \\
& q_{3}(\bar{t})=\bar{a}_{3} \cos \left(\bar{\Omega} \bar{t}-\gamma_{3}\right) .
\end{aligned}
$$

$\left\{\bar{a}_{i}\right\}_{i=1,2,3}$ and $\left\{\gamma_{i}\right\}_{i=1,2,3}$ are the dimensionless amplitudes and phase differences of the three modal coordinates, analytically obtained in [7] as functions of forcing $Q$ and excitation frequency $\bar{\Omega}$. Their analytical expressions are recalled in Appendix 8. In [7], it is shown that only three stable vibratory solutions can be obtained in the steady state:

- the SDOF solution (single-degree-of-freedom), with $\bar{a}_{1} \equiv \bar{a}_{2} \equiv 0$ and $\bar{a}_{3} \neq 0$, which is the usual uncoupled solution;

- the $\mathrm{C}_{1}$ solution, with $\bar{a}_{1} \neq 0, \bar{a}_{2} \equiv 0, \bar{a}_{3} \neq 0$ : an energy transfer occurs between modes 3 and 1 ;

- the $\mathrm{C}_{2}$ solution, with $\bar{a}_{1} \equiv 0, \bar{a}_{2} \neq 0, \bar{a}_{3} \neq 0$ : an energy transfer occurs between modes 3 and 2 . 
Fig. 8 Theoretical frequency response curves. (Upper view) Amplitudes as a function of $\bar{\Omega}$. (Lower view) Phases as a function of $\bar{\Omega}$

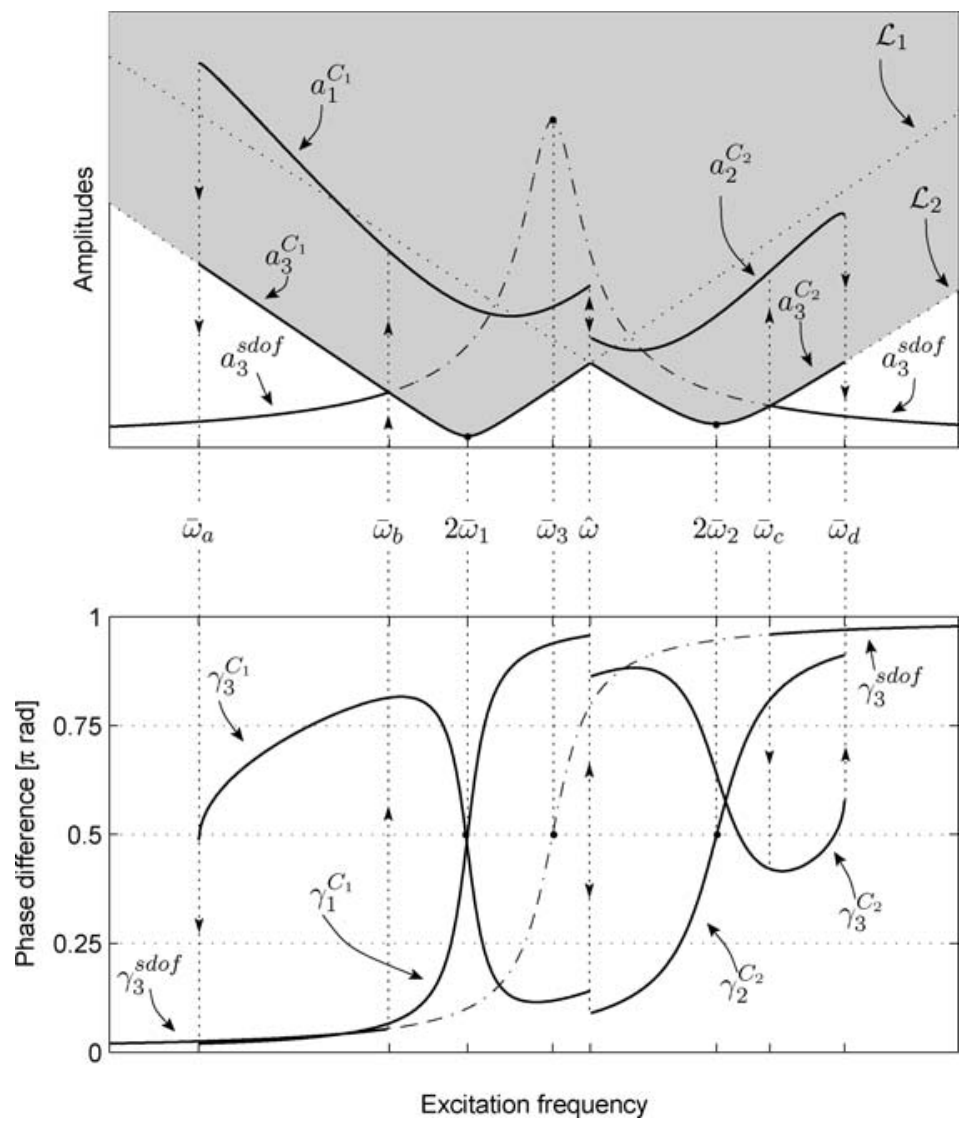

It is also theoretically proved that a stable fourth solution with $\bar{a}_{1} \neq 0, \bar{a}_{2} \neq 0$, and $\bar{a}_{3} \neq 0$ can be obtained only if the structure is perfectly axisymmetric, that is to say if $\bar{\omega}_{1}=\bar{\omega}_{2}, \alpha_{1}=\alpha_{2}$, and $\xi_{1}=\xi_{2}$. This case is unrealistic in practice, as a real structure (and especially the shell under study) always owes imperfections that lead to different values of parameters for the companion modes.

\subsection{Theoretical frequency response curves}

In the $\left(\bar{a}_{3}, \bar{\Omega}\right)$ plane, the SDOF solution is stable outside an instability region, inside which the coupled solutions $\mathrm{C}_{1}$ and $\mathrm{C}_{2}$ originate. This region is shown with a gray shaded area in Fig. 8. It is then possible to predict which solution ( $\mathrm{SDOF}, \mathrm{C}_{1}$, or $\mathrm{C}_{2}$ ) is obtained by observing the position of the SDOF solution with respect to the instability region [7].

Figure 8 shows the values of $\left\{\left(\bar{a}_{i}, \gamma_{i}\right)\right\}_{i=1,2,3}$ as functions of $\bar{\Omega}$ for a particular value of $Q$, when $\bar{\Omega}$ is close to $\bar{\omega}_{3}$. In Fig. 8 , for each couple of amplitude and phase solution, the subscripts denote the number of the mode (mode 1,2, or 3) and the superscripts refer to the solution branch (SDOF, $\mathrm{C}_{1}$, or $\mathrm{C}_{2}$ ). One recalls that the values of $\bar{a}_{1}$ (resp. $\bar{a}_{2}$ ) for the SDOF and $\mathrm{C}_{2}$ (resp. $\mathrm{C}_{1}$ ) solutions are zero and are not shown in Fig. 8. The analytical expressions of $\left\{\gamma_{i}\right\}_{i=1,2,3}$, not included in [7], can be found in Appendix 8.

A few remarkable properties of the frequency response curves are now enumerated.

- Solution branches $a_{3}^{C_{1}}$ and $a_{3}^{C_{2}}$ coincides with the limits $\mathcal{L}_{1}$ and $\mathcal{L}_{2}$ of the instability regions.

- The frequencies of the minima of $\mathcal{L}_{1}$ and $\mathcal{L}_{2}$ correspond respectively to $2 \bar{\omega}_{1}$ and $2 \bar{\omega}_{2}$, twice the natural frequencies of modes 1 and 2 . In these cases, phases $\gamma_{1}^{C_{1}}$ and $\gamma_{2}^{C_{2}}$ have the particular value $\pi / 2$.

- More than one stable solution coexists in some frequency intervals. In the case of Fig. 8, SDOF and $\mathrm{C}_{1}$ solutions coexist in $\left[\bar{\omega}_{a}, \bar{\omega}_{b}\right]$ and $\mathrm{SDOF}$ and $\mathrm{C}_{2}$ solutions coexist in $\left[\bar{\omega}_{c}, \bar{\omega}_{d}\right]$. The SDOF solution is stable for $\bar{\Omega}<\bar{\omega}_{a}$ or $\bar{\Omega}>\bar{\omega}_{d}, \mathrm{C}_{1}$ solution is stable only in $\left[\bar{\omega}_{b}, \hat{\omega}\right]$ and $\mathrm{C}_{2}$ solution is stable only in $\left[\hat{\omega}, \bar{\omega}_{d}\right]$. As 


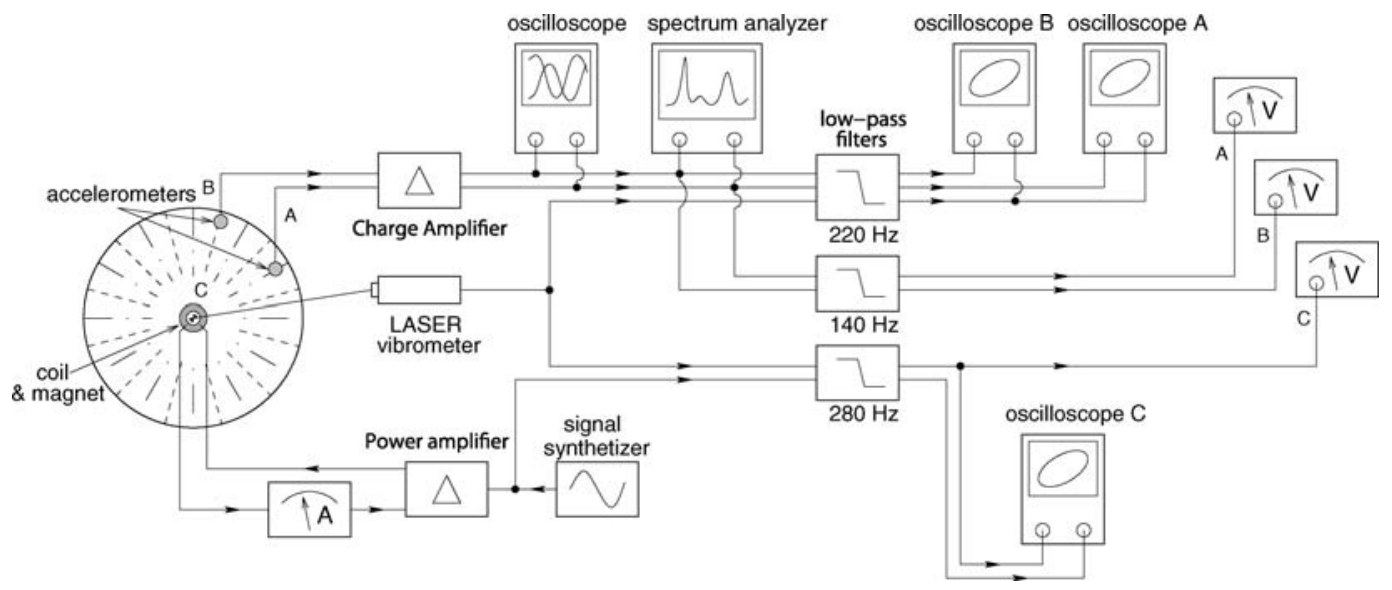

Fig. 9 Experimental setup for measuring the frequency response curves

a consequence, depending on the initial conditions, jump phenomena can occur at frequencies $\bar{\omega}_{a}$ and $\bar{\omega}_{b}$ between SDOF and $\mathrm{C}_{1}$ solutions, at frequency $\hat{\omega}$ between $\mathrm{C}_{1}$ and $\mathrm{C}_{2}$ solutions, and at $\bar{\omega}_{c}$ and $\bar{\omega}_{d}$ between SDOF and $\mathrm{C}_{2}$ solutions.

- $\hat{\omega}$ denotes the frequency where $\mathcal{L}_{1}$ and $\mathcal{L}_{2}$ meet. A stability exchange occurs between $\mathrm{C}_{1}$ and $\mathrm{C}_{2}$ solution at this frequency, if the SDOF solution is inside the instability region at this frequency. As a consequence, there is no frequency interval where $\mathrm{C}_{1}$ and $\mathrm{C}_{2}$ solutions coexist.

\section{Measurements of the 1:1:2 internal resonance}

In this section, the special procedures that lead to the measurements of a particular 1:1:2 internal resonance on the shell introduced in Sections 2 and 3 are described. It occurs between mode $(0,1)$ (denoted in the following by mode 3 , of natural frequency $f_{3}$ close to $225 \mathrm{~Hz})$ and with both companion modes $(0,6)$ (denoted in the following by mode 1 and mode 2 , of natural frequencies $f_{1}$ and $f_{2}$ close to 110 and $111 \mathrm{~Hz}$, see Table 2).

\subsection{Experimental setup}

The experimental setup is shown in Figs. 9 and 10. The measuring devices are listed in Table 3.

The location of the magnet for the excitation is chosen at the center of the shell, so that only the axisymmetric mode (mode 3 ) is directly excited. The driving signal is chosen simple-harmonic, with its frequency $f_{\mathrm{dr}}$ chosen in the vicinity of the natural frequency $f_{3}$ of mode 3 .

Three transducers (two accelerometers and a laser vibrometer) are used for measuring the time evolutions of the three involved modes. The laser vibrometer beam points the center of the shell, allowing measurement of mode 3 time evolution only. Accelerometer $A$ (resp. $B$ ) is precisely located on a node of mode 2 (resp. mode 1 ), so that it measures the time evolution of mode 1 (resp. mode 2) only. To check the location of the accelerometers, the shell is subjected to an acoustic noise excitation and the frequency response functions of the accelerometer signals, with respect to the acoustic pressure, are measured. Figure 11 is obtained and one can verify that only one peak is obtained for each accelerometer. For accelerometer $A$, the peak frequency is around $110 \mathrm{~Hz}$, the natural frequency of mode 1 ; and for accelerometer $\mathrm{B}$, the peak frequency is around $111 \mathrm{~Hz}$, the natural frequency of mode 2 .

The three signals are filtered so that only their fundamental component (of frequency $f_{\mathrm{dr}} / 2$ for modes 1 and 2 and $f_{\text {dr }}$ for mode 3 ) is retained. This operation

Table 3 Devices used during the measurement of the frequency response curves

\begin{tabular}{lll}
\hline Accelerometers & Brüel \& Kjær & 4375 \\
Charge amplifier & Brüel \& Kjær & NEXUS \\
Laser vibrometer & $\begin{array}{l}\text { Polytec } \\
\text { Filters }\end{array}$ & $\begin{array}{l}\text { OFV 303 / OFV 3001 S } \\
\text { systems }\end{array}$ \\
$\begin{array}{c}\text { Sine and noise } \\
\text { synthesizer }\end{array}$ & Brüel \& Kjær & 1049 \\
$\begin{array}{c}\text { Power amplifier } \\
\text { Brüel \& Kjær }\end{array}$ & 2706 \\
\hline
\end{tabular}


Fig. 10 (a) Fixation of the shell with nylon threads; location of accelerometers A and B, respectively, on nodal diameters of modes 2 and 1 , location of the magnet and the laser beam at the center of the shell. (b) Measured mode shape of mode 3 , in RMS amplitude. (c) and (d) Measured mode shapes of companion asymmetric modes 1 and 2, in RMS amplitude
Fig. 11 Frequency response function of accelerometer $A$ and $B$ with respect to an acoustic pressure excitation

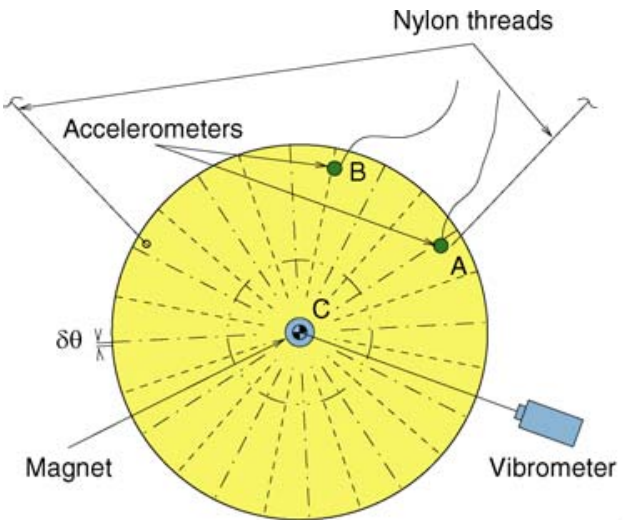

(a)

Nylon threads

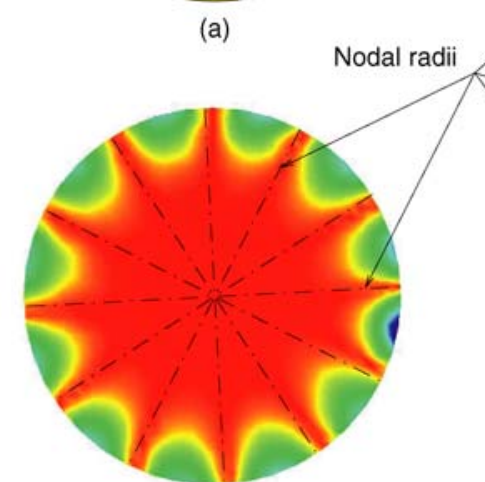

(c) - mode 1, (6,0), $110 \mathrm{~Hz}$

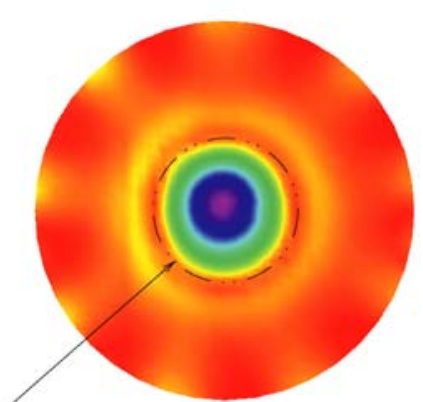

(b) - mode 3, $(0,1), 220 \mathrm{~Hz}$

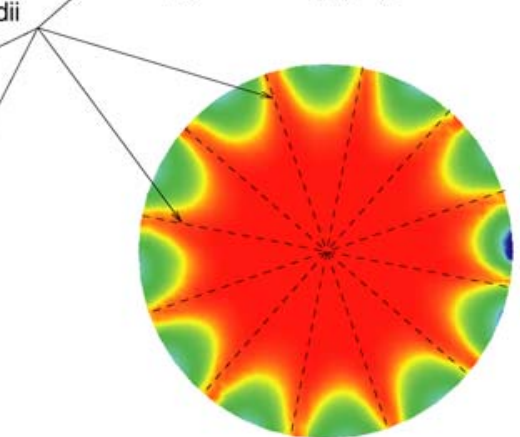

(d) - mode 2, (6,0), $111 \mathrm{~Hz}$

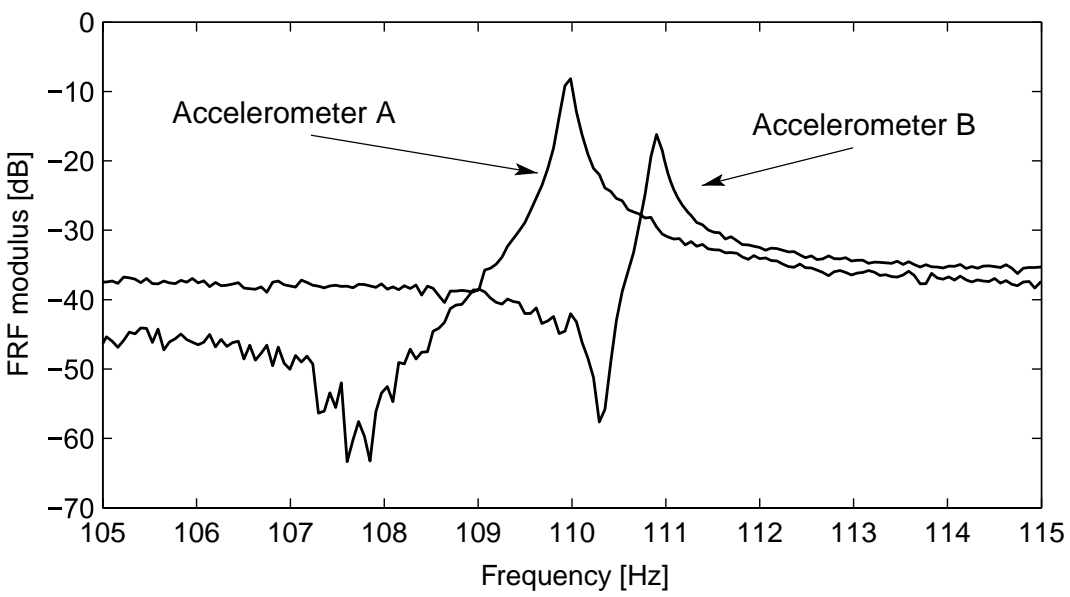

also eliminates the slight contribution of mode 3 to the accelerometer signals, since points $A$ and $B$ are not located on the nodal circle of mode 3 . The cutoff frequencies of the filters are adjusted so that the fundamental component of the signals is not altered. This filtering operation is needed because we want to compare the present experimental results to the theoretical ones described in Section 4, which were obtained with a first-order perturbative development. Then, the root mean square (RMS) values of the amplitudes of the three filtered signals are measured by voltmeters.

Two oscilloscopes (named A and B in Fig. 9) are used to measure the phase differences of the accelerometer signals with respect to the vibrometer signal to estimate the values of $\gamma_{1}$ and $\gamma_{2}$ in Equations (8a) and (8b). As the fundamental frequencies of the signals are not 
identical, a special procedure, detailed in Appendix C, is used to estimate $\gamma_{1}$ and $\gamma_{2}$. Phase $\gamma_{3}$ is measured in a straightforward manner by using oscilloscope $\mathrm{C}$ to measure the time delay of the vibrometer signal with respect to the force signal. Both signals are processed by identical filters to cancel the influence of the phase differences between the input and the output of the filters. The force signal used here is in fact a signal proportional to the current intensity in the coil, obtained by the terminal voltage of the ammeter. As it is shown in [14], this signal can be considered to be proportional to the force acting on the structure, with a negligible frequency dependence, and thus in phase with it.

Considering the properties of the frequency response curves presented in Section 4.2, it appears that easier observation of both coupled solutions $\mathrm{C}_{1}$ and $\mathrm{C}_{2}$ are obtained if the natural frequency $f_{3}$ of mode 3 is located between $2 f_{1}$ and $2 f_{2}$. For this reason, a small ballast is glued with beeswax at the center of the shell in order to slightly lower $f_{3}$. A ballast of $7.5 \mathrm{~g}$ was used for the experiments discussed in Section 6 to lower $f_{3}$ from approximately 225 to $220 \mathrm{~Hz}$. It has been verified that the addition of this ballast does not change the values of $f_{1}$ and $f_{2}$, since the corresponding mode shapes have nodes at the center of the shell.

\subsection{Processing the signals}

According to the model described in Section 4, the dimensionless displacements of the shell at the measuring points $A, B$, and $C$ (Fig. 10) writes:

$$
\begin{aligned}
\bar{w}_{A}(\bar{t})= & R_{60}\left(\bar{r}_{m}\right) \cos 6 \delta \theta \bar{a}_{1} \cos \left(\frac{\bar{\Omega}}{2} \bar{t}-\frac{\gamma_{1}+\gamma_{3}}{2}\right) \\
& +R_{01}\left(\bar{r}_{m}\right) \bar{a}_{3} \cos \left(\bar{\Omega} \bar{t}-\gamma_{3}\right) \\
\bar{w}_{B}(\bar{t})= & R_{60}\left(\bar{r}_{m}\right) \bar{a}_{2} \cos \left(\frac{\bar{\Omega}}{2} \bar{t}-\frac{\gamma_{2}+\gamma_{3}}{2}\right) \\
& +R_{01}\left(\bar{r}_{m}\right) \bar{a}_{3} \cos \left(\bar{\Omega} \bar{t}-\gamma_{3}\right), \\
\bar{w}_{C}(\bar{t})= & R_{01}(0) \bar{a}_{3} \cos \left(\bar{\Omega} \bar{t}-\gamma_{3}\right),
\end{aligned}
$$

where the mode shapes have been written as:

$$
\begin{aligned}
& \Phi_{1}(\bar{r}, \theta)=R_{60}(\bar{r}) \cos (6 \theta), \\
& \Phi_{2}(\bar{r}, \theta)=R_{60}(\bar{r}) \sin (6 \theta+6 \delta \theta), \\
& \Phi_{3}(\bar{r}, \theta)=R_{01}(\bar{r}) .
\end{aligned}
$$

In the previous equations, $\bar{r}_{m}$ denotes the common radius of locations of the accelerometers (points $A$ and $B),\left\{\bar{a}_{i}\right\}_{i=1,2,3}$ and $\left\{\gamma_{i}\right\}_{i=1,2,3}$ are the dimensionless amplitudes and phases introduced in Equation (8), $R_{60}(\bar{r})$ and $R_{01}(\bar{r})$ are the radial part of the mode shapes. $\delta \theta$ represents a small angle introduced here to take into account the slight imperfections of the geometry of the shell. Those imperfections are responsible for an angular shift in the location of the nodal diameters of the companion asymmetric modes with respect to the perfect case where the nodes of one of the companion modes are located exactly midway between the nodes of the other $[14,16]$. However, in our case, $\delta \theta \simeq 1^{\circ}$ and $\cos 6 \delta \theta=0.9945 \simeq 1$ so that this quantity will be neglected in the following.

According to the filtering operations, Equation (9) becomes

$$
\begin{aligned}
& w_{A}(t)=a_{1} \cos \left(2 \pi \frac{f_{\mathrm{dr}}}{2} t-\frac{\gamma_{1}+\gamma_{3}}{2}\right), \\
& w_{B}(t)=a_{2} \cos \left(2 \pi \frac{f_{\mathrm{dr}}}{2} t-\frac{\gamma_{2}+\gamma_{3}}{2}\right), \\
& w_{C}(t)=a_{3} \cos \left(2 \pi f_{\mathrm{dr}} t-\gamma_{3}\right),
\end{aligned}
$$

where $t$ is the time, $\left\{w_{i}\right\}_{i=A, B, C}$ denotes the dimensioned displacements of points $A, B$, and $C$ and $\left\{a_{i}\right\}_{i=A, B, C}$ their amplitudes. The three measured signals and the force signal can be written as:

$$
\begin{aligned}
& s_{A}(t)=\sqrt{2} s_{A}^{0} \cos \left(\pi f_{\mathrm{dr}} t-\varphi_{A}\right), \\
& s_{B}(t)=\sqrt{2} s_{B}^{0} \cos \left(\pi f_{\mathrm{dr}} t-\varphi_{B}\right), \\
& s_{C}(t)=\sqrt{2} s_{C}^{0} \cos \left(2 \pi f_{\mathrm{dr}} t-\varphi_{C}\right), \\
& s_{F}(t)=\sqrt{2} s_{F}^{0} \cos \left(2 \pi f_{\mathrm{dr}}\right),
\end{aligned}
$$

where $\left\{s_{i}^{0}\right\}_{i=A, B, C}$ are the three measured RMS amplitudes, $s_{F}^{0}$ the RMS amplitude of the force signal, and $\left\{\varphi_{i}\right\}_{i=A, B, C}$ are the phases differences with respect to the force signal. Bearing in mind that $s_{A}$ and $s_{B}$ are acceleration signals and that $s_{C}$ is a velocity signal and by assuming that those signals are pure sine functions, the identification between Equations (11) and (12) 
gives:

$a_{1}=\sqrt{2} \frac{s_{A}^{0}}{\pi^{2} f_{\mathrm{dr}}^{2}}, \quad a_{2}=\sqrt{2} \frac{s_{B}^{0}}{\pi^{2} f_{\mathrm{dr}}^{2}}$,

$a_{3}=\sqrt{2} \frac{s_{C}^{0}}{2 \pi f_{\mathrm{dr}}}$,

$\gamma_{3}=\varphi_{C}+\pi / 2$.

Phases $\gamma_{1}$ and $\gamma_{2}$ are obtained by a procedure described in Appendix C.

The dimensioned force acting at the center of the structure can be written as:

$F(t)=F_{\mathrm{dr}} \cos \left(2 \pi f_{\mathrm{dr}} t\right), \quad$ with $\quad F_{\mathrm{dr}}=K \sqrt{2} I$,

where $I$ is the RMS amplitude of the current intensity in the coil, obtained with the ammeter. In this study, coefficient $K$ is estimated by adjusting the parameters of the theoretical model, so that theory and experiment match in the case of a low $F_{\mathrm{dr}}$ and thus of a linear frequency response curve. The value $K=0.653 \mathrm{~N} / \mathrm{A}$ was found (Section 7).

\subsection{Dimensioned parameters}

In order to compare the experimental results with the theoretical results, the relations between the dimensionless parameters introduced in Section 4, and their dimensioned counterpart are given here, from [7]. One has:

$$
r=a \bar{r}, \quad t=\frac{a^{2}}{h} \sqrt{\frac{12 \rho\left(1-v^{2}\right)}{E}} \bar{t},
$$

$w(r, \theta, t)=\frac{h^{3}}{a^{2}} \bar{w}(\bar{r}, \theta, \bar{t})$,

$$
\begin{aligned}
a_{1} & =\frac{h^{3}}{a^{2}} R_{60}\left(\bar{r}_{m}\right) \bar{a}_{1}, \quad a_{2}=\frac{h^{3}}{a^{2}} R_{60}\left(\bar{r}_{m}\right) \bar{a}_{2}, \\
a_{3} & =\frac{h^{3}}{a^{2}} R_{01}(0) \bar{a}_{3}, \\
\varepsilon & =12\left(1-v^{2}\right) \frac{h}{R}, \quad F_{\mathrm{dr}}=\frac{1}{R_{01}(0)} \frac{E h^{7}}{R a^{4}} Q .
\end{aligned}
$$

The analogous relation for the frequencies is given by Equation (1).

\section{Experimental results}

In this section, three sets of experimental results are exposed. Firstly, some frequency response curves are shown. Then, the measurement of the instability region is described. Finally, the vibratory patterns of the shell at the driving frequency as well as at half the driving frequency are shown for the coupled regimes $C_{1}$ and $\mathrm{C}_{2}$.

\subsection{Frequency response curves}

Frequency response curves have been obtained by holding constant the amplitude $F_{\mathrm{dr}}$ of the excitation and measuring the amplitudes $a_{1}, a_{2}$, and $a_{3}$, for various frequencies $f_{\mathrm{dr}}$ of excitations, during step-bystep forward and backward sweeps. Figure 12 gathers the theoretical SDOF solutions corresponding to the four selected values of the excitation amplitude $F_{\text {dr }} \in\{0.0277,0.138,0.556,1.108\} \mathrm{N}$ as well as the instability region. This figure is useful to predict the occurrence of the coupled solutions, by observing the
Fig. 12 Theoretical SDOF frequency response curve and instability region for four different forcing levels. (-) $a_{3}$ stable. (--) $a_{3}$ unstable. (1)

$F_{\text {dr }}=0.0277 \mathrm{~N}$ (Fig. 13),

(2) $F_{\mathrm{dr}}=0.138 \mathrm{~N}$ (Fig. 14),

(3) $F_{\mathrm{dr}}=0.556 \mathrm{~N}$ (Fig. 15),

(4) $F_{\mathrm{dr}}=1.108 \mathrm{~N}$ (Fig. 16).

$\alpha_{1}=0.75, \alpha_{2}=0.7$,

$\xi_{1}=5 \times 10^{-4}$,

$\xi_{2}=6 \times 10^{-4}$,

$\xi_{3}=6.5 \times 10^{-4}$

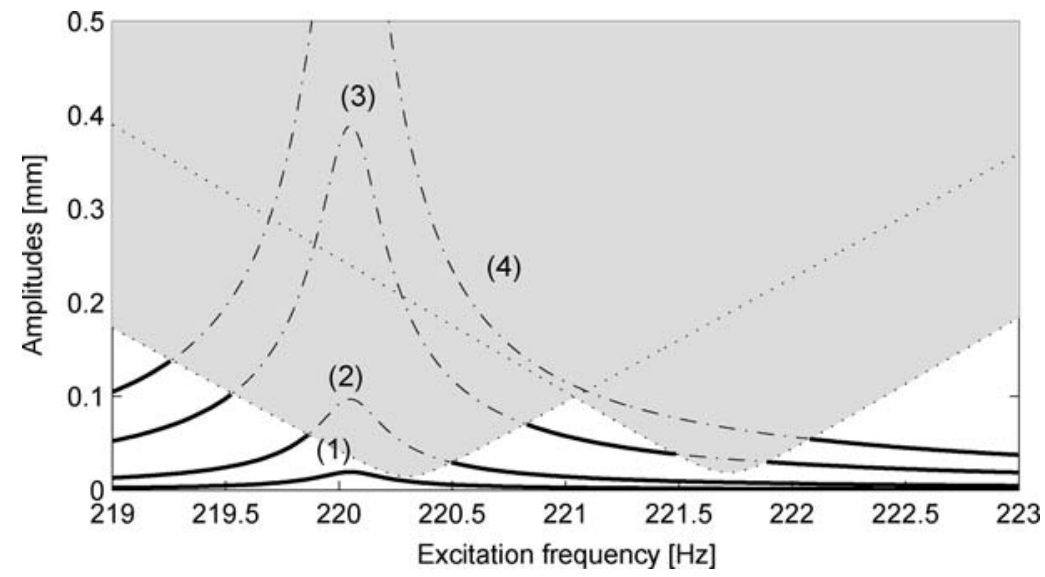




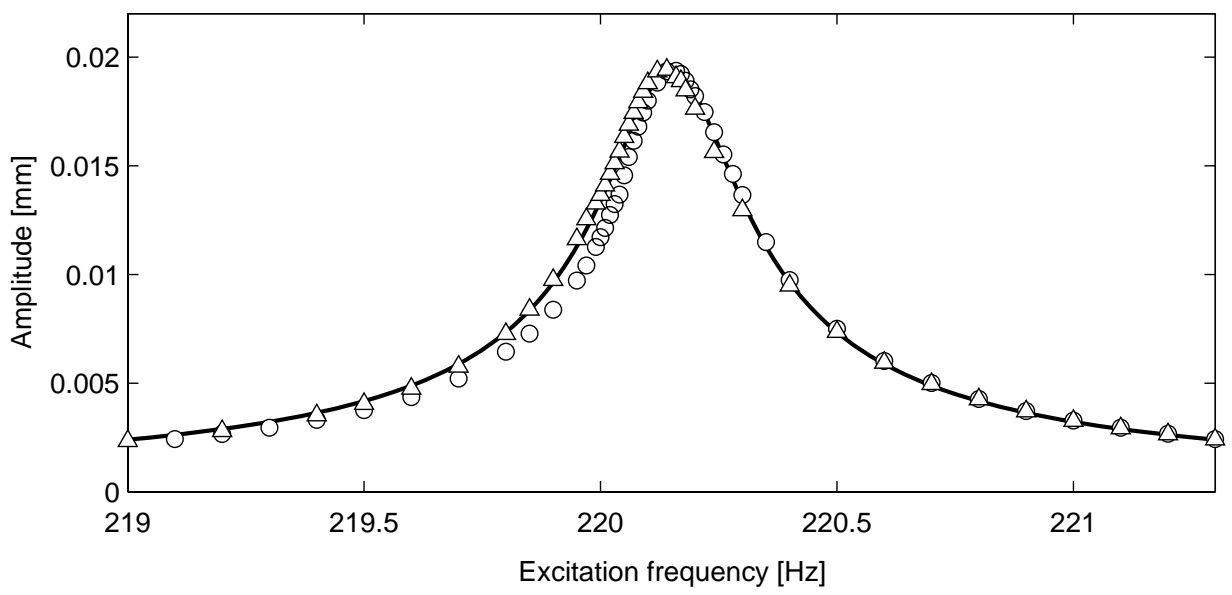

Fig. 13 Frequency response curve for $F_{\mathrm{dr}}=0.0277 \mathrm{~N}$ (Experience (1)). (-) $a_{3}$ from theory. (o) $a_{3}$ obtained with an increasing frequency sweep. $(\triangle) a_{3}$ obtained with a decreasing frequency sweep
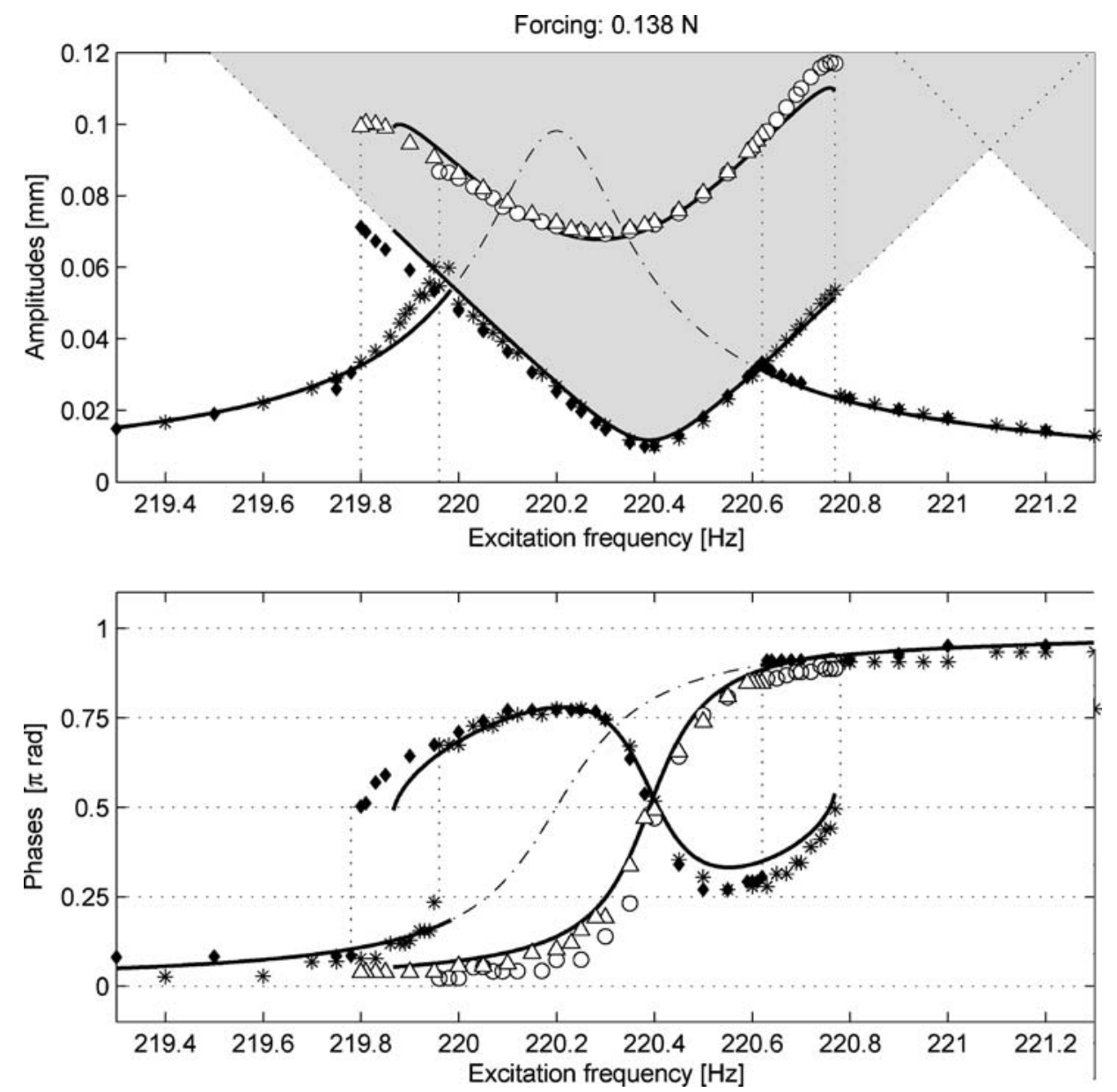

Fig. 14 Frequency response curve, for $F_{\mathrm{dr}}=0.138 \mathrm{~N}$ (Experience (2)), (一) $a_{1}, \gamma_{1}, a_{3}, \gamma_{3}$ from theory, (०) $a_{1}$ and $\gamma_{1},(*) a_{3}$ and $\gamma_{3}$, experiments with an increasing frequency sweep, $(\Delta) a_{1}$ and $\gamma_{1},(\diamond) a_{3}$ and $\gamma_{3}$, experiments with a decreasing frequency sweep 


\section{Forcing: $0.554 \mathrm{~N}$}
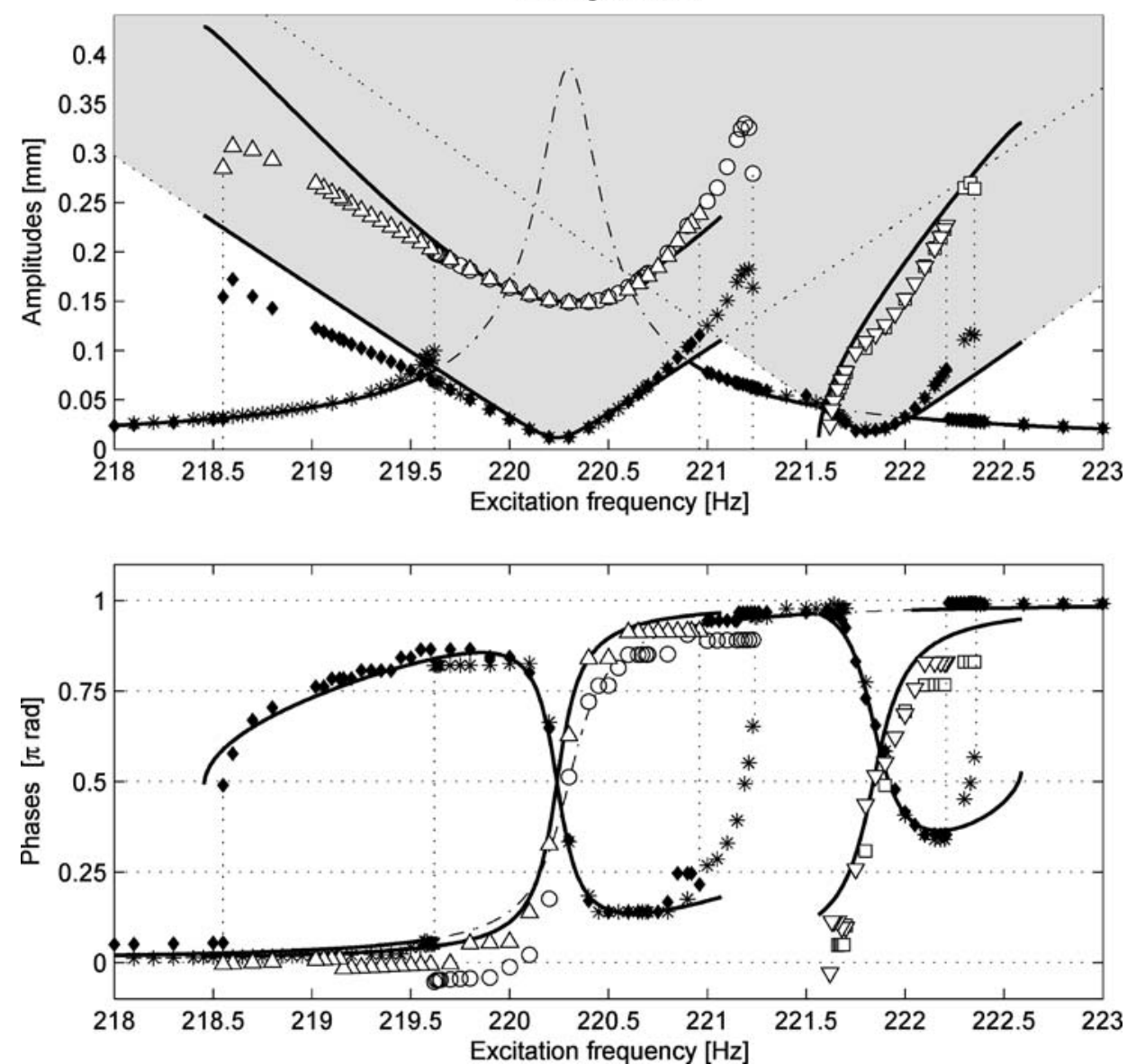

Fig. 15 Frequency response curve, for $F_{\mathrm{dr}}=0.556 \mathrm{~N}$ (Experience (3)), (-) $a_{1}, \gamma_{1}, a_{2}, \gamma_{2}, a_{3}$, and $\gamma_{3}$ from theory, (о) $a_{1}$ and $\gamma_{1},(\square) a_{2}$ and $\gamma_{2},(*) a_{3}$ and $\gamma_{3}$, experiments with an increasing

position of the SDOF curves with respect to the instability regions. The four corresponding experimental frequency response curves are shown in Figs. 13-16, along with the corresponding theoretical curves that are discussed in Section 7. Those forcing conditions have been selected so that remarkable nonlinear vibratory regimes are obtained.

- For $F_{\mathrm{dr}}=0.0277 \mathrm{~N}$ (Experience (1), Fig. 13), no coupled solution is obtained as the SDOF curve does not enter the instability region. It has the shape of the usual frequency response curve of a linear 1-degreeof-freedom system.

- For $F_{\mathrm{dr}}=0.138 \mathrm{~N}$ (Experience (2), Fig. 14), the SDOF solution enters only the $\mathcal{L}_{1}$ boundary of the instability region so that only a $\mathrm{C}_{1}$ solution can be observed. frequency sweep, $(\Delta) a_{1}$ and $\gamma_{1},(\nabla) a_{2}$ and $\gamma_{2},(\diamond) a_{3}$ and $\gamma_{3}$, experiments with a decreasing frequency sweep

- For $F_{\mathrm{dr}}=0.556 \mathrm{~N}$ (Experience (3), Fig. 15), the SDOF solution enters both $\mathcal{L}_{1}$ and $\mathcal{L}_{2}$ boundaries at two distinct locations, so that the SDOF solution for $f_{\mathrm{dr}}=\hat{f}$ (where $\hat{f}$ is the frequency of the meeting point of $\mathcal{L}_{1}$ and $\mathcal{L}_{2}$, see Fig. 8) is stable. As a consequence, with an increasing frequency sweep, one obtains the following succession of solutions: SDOF, $\mathrm{C}_{1}, \mathrm{SDOF}, \mathrm{C}_{2}$, SDOF.

- For $F_{\mathrm{dr}}=1.108 \mathrm{~N}$ (Experience (4), Fig. 16), the forcing level is large enough so that the SDOF solution enters both $\mathcal{L}_{1}$ and $\mathcal{L}_{2}$ boundaries and is unstable for $f_{\mathrm{dr}}=\hat{f}$. Thus, an exchange of stability between $\mathrm{C}_{1}$ and $\mathrm{C}_{2}$ solution occurs around $f_{\mathrm{dr}}=\hat{f}$ and with an increasing frequency sweep, one obtains the following succession of solutions: SDOF, $\mathrm{C}_{1}, \mathrm{C}_{2}$, SDOF. 


\section{Forcing: $1.108 \mathrm{~N}$}
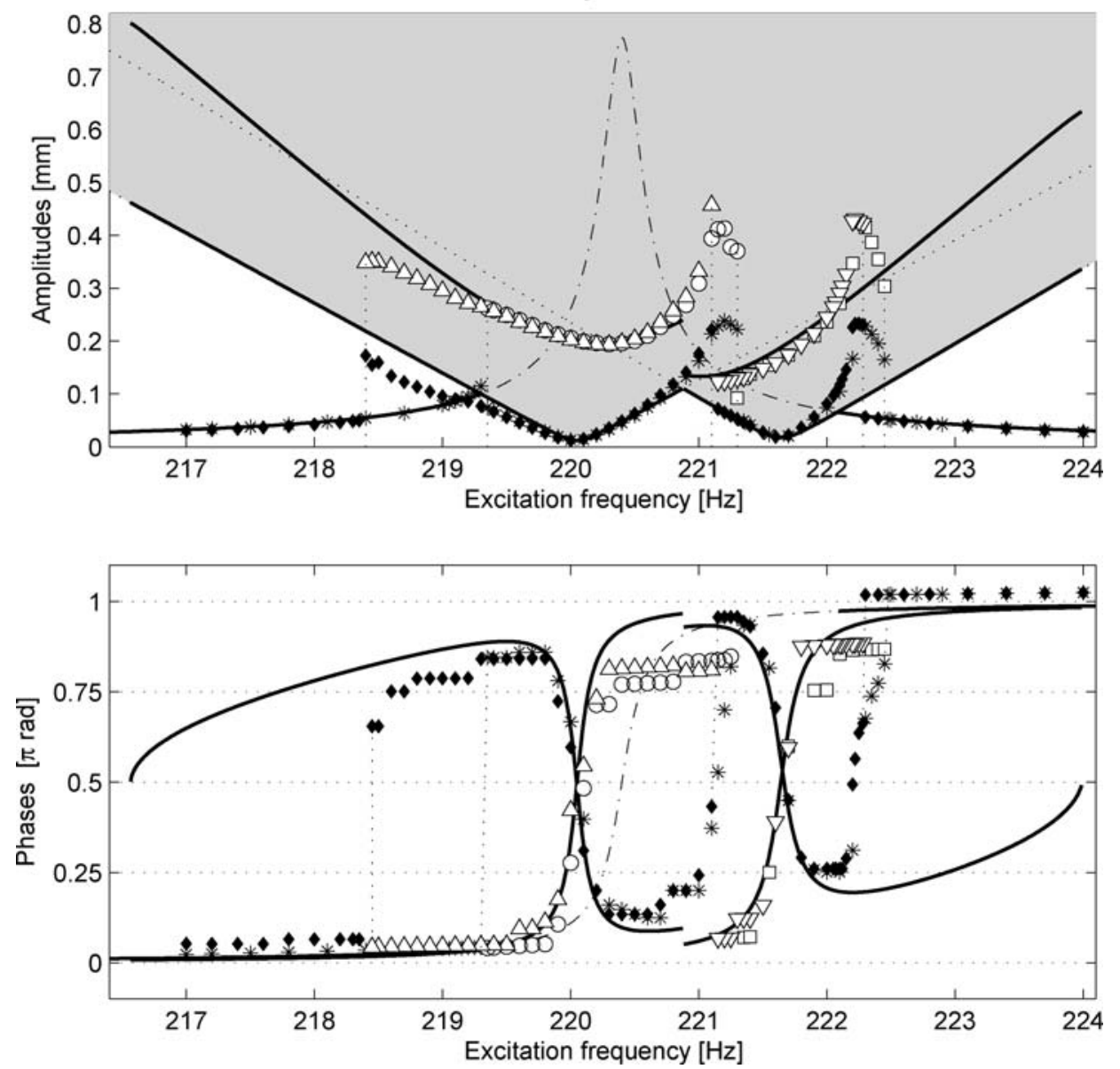

Fig. 16 Frequency response curve, for $F_{\mathrm{dr}}=1.108 \mathrm{~N}$ (Experience (4)), (-) $a_{1}, \gamma_{1}, a_{2}, \gamma_{2}, a_{3}$, and $\gamma_{3}$ from theory, (०) $a_{1}$ and $\gamma_{1},(\square) a_{2}$ and $\gamma_{2},(*) a_{3}$ and $\gamma_{3}$, experiments with an increasing frequency sweep, $(\Delta) a_{1}$ and $\gamma_{1},(\nabla) a_{2}$ and $\gamma_{2},(\diamond) a_{3}$ and $\gamma_{3}$, experiments with a decreasing frequency sweep
Fig. 17 Boundary of the instability region. (-) Theory. (o) Experiments obtained with an increasing frequency sweep. $(\Delta)$

Experiments obtained with a decreasing frequency sweep

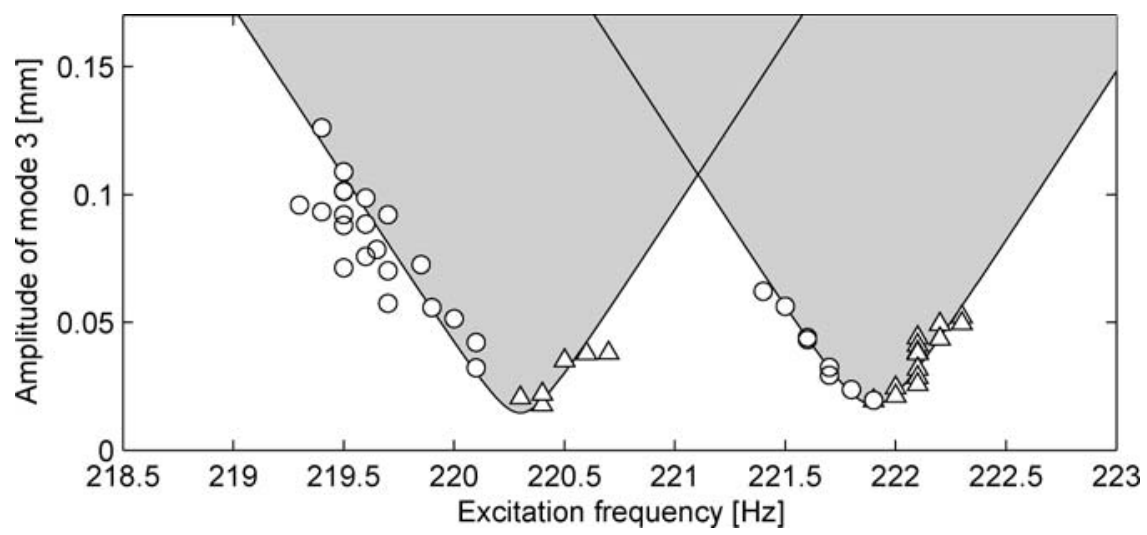


Fig. 18 RMS velocity amplitude of the shell during a $\mathrm{C}_{1}$ coupling, obtained with $f_{\mathrm{dr}}=218.3 \mathrm{~Hz}$ and $F_{\mathrm{dr}}=1.5 \mathrm{~N}$

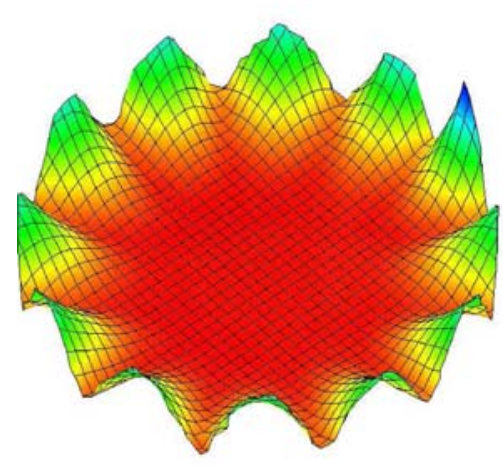

Frequency $f_{d r} / 2$

Fig. 19 RMS velocity amplitude of the shell during a $\mathrm{C}_{2}$ coupling obtained with $f_{\mathrm{dr}}=222.8 \mathrm{~Hz}$ and $F_{\mathrm{dr}}=1.5 \mathrm{~N}$

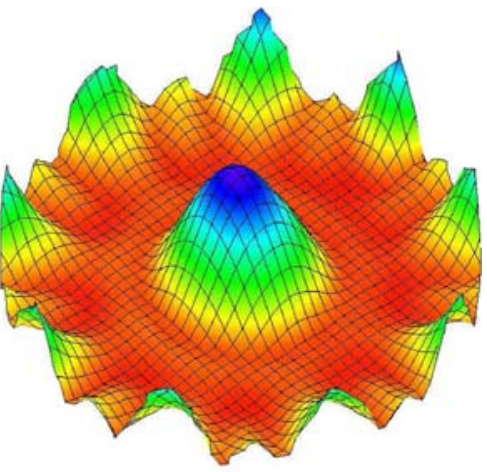

Frequency $f_{d r}$

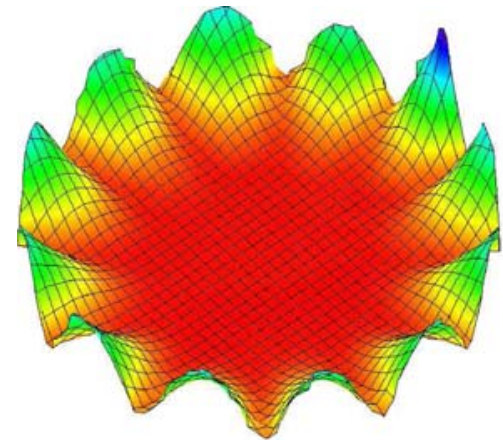

Frequency $f_{d r} / 2$

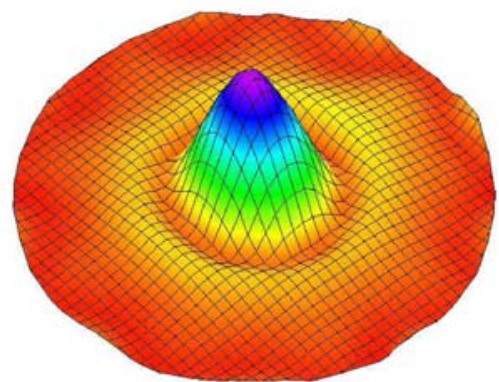

Frequency $f_{d r}$

\subsection{Instability region}

The boundaries $\mathcal{L}_{1}$ and $\mathcal{L}_{2}$ of the instability region can be estimated experimentally by the following procedure: each point, related to a particular amplitude of excitation, is obtained by measuring amplitude $a_{3}$ and excitation frequency $f_{\mathrm{dr}}$ at the precise location where the SDOF solution becomes unstable and coupled solutions arise $\left(\mathrm{C}_{1}\right.$ to obtain $\mathcal{L}_{1}$ and $\mathrm{C}_{2}$ to obtain $\left.\mathcal{L}_{2}\right)$. This measurement is realized by sweeping forward and backward in frequency, and is repeated for different forcing levels. The obtained results are shown in Fig. 17.

\subsection{Deflection shape in the nonlinear regime}

The nonlinear vibratory regimes corresponding to $\mathrm{C}_{1}$ and $C_{2}$ solutions can be visualized with a scanning laser vibrometer. The structure is driven at center with a sine force signal, with $F_{\mathrm{dr}}$ and $f_{\mathrm{dr}}$ chosen so that $\mathrm{C}_{1}$ or $\mathrm{C}_{2}$ solutions are reached. The velocity of the structure is measured in each point of a $40 \times 40$ grid. Due to the presence of the coupling with modes 1 and 2, the signals are periodic of frequency $f_{\mathrm{dr}} / 2$. Figures 18 and 19 show the deflection shapes at frequencies $f_{\mathrm{dr}} / 2$ (the subharmonic) and $f_{\mathrm{dr}}$ (the excitation frequency), obtained by calculating a fast Fourier transform of the measured signals. One can observe that the deflection shape at the subharmonic frequency is mainly governed by the mode shapes of mode 1 for $\mathrm{C}_{1}$ coupling and mode 2 for $\mathrm{C}_{2}$ coupling. Moreover, the deflection shape at the excitation frequency $f_{\mathrm{dr}}$ is mainly composed by mode 3 deflection shape for $\mathrm{C}_{2}$ coupling. For $\mathrm{C}_{1}$ coupling, some harmonics of mode 1 as well as a linear coupling with mode $(9,0)$ (of natural frequency $214 \mathrm{~Hz}$ ) probably explains the deformation of the shape near the boundary of the shell. As these figures show the RMS values of the deflection shapes, all extrema are positive. As a consequence, all anti-nodes are upward, in contrast to Fig. 6 in which as usual, for two successive anti-nodes, one is upward and the other is downward. 
Table 4 Model parameters obtained theoretically in [7] (first column) and by fitting the theoretical frequency response curves to the experimental ones, for various experiments

\begin{tabular}{|c|c|c|c|c|c|c|c|}
\hline & Theory & $\begin{array}{l}\text { Modal } \\
\text { analysis }\end{array}$ & $\begin{array}{l}\text { Experience (1) } \\
\text { (Fig. 13) }\end{array}$ & $\begin{array}{l}\text { Experience (2) } \\
\text { (Fig. 14) }\end{array}$ & $\begin{array}{l}\text { Experience (3) } \\
\text { (Fig. 15) }\end{array}$ & $\begin{array}{l}\text { Experience (4) } \\
\text { (Fig. 16) }\end{array}$ & $\begin{array}{l}\text { Instability region } \\
\text { (Fig. 17) }\end{array}$ \\
\hline$K(\mathrm{~N} / \mathrm{A})$ & - & - & 0.653 & 0.653 & 0.653 & 0.653 & - \\
\hline$f_{1}(\mathrm{~Hz})$ & 101.77 & 110.1 & - & 110.20 & 110.12 & 110.03 & 110.15 \\
\hline$f_{2}(\mathrm{~Hz})$ & 101.77 & 110.86 & - & - & 110.92 & 110.82 & 110.95 \\
\hline$f_{3}(\mathrm{~Hz})$ & 386.03 & 220.05 & 220.15 & 220.2 & 220.3 & 220.4 & 220.2 \\
\hline$\xi_{1}\left(10^{-4}\right)$ & - & 4.5 & - & 4 & 4 & 4 & 5 \\
\hline$\xi_{2}\left(10^{-4}\right)$ & - & 5.4 & - & - & 5.5 & 5.5 & 6 \\
\hline$\xi_{3}\left(10^{-4}\right)$ & - & 6.7 & 6.5 & 6.4 & 6.5 & 6.5 & 6.4 \\
\hline$\alpha_{1}$ & 30 & - & - & 0.75 & 0.75 & 0.75 & 0.75 \\
\hline$\alpha_{2}$ & 30 & - & - & - & 0.7 & 0.7 & 0.75 \\
\hline$\alpha_{3}$ & 13.8 & - & - & 0.95 & 0.95 & 1.1 & - \\
\hline$\alpha_{4}$ & 13.8 & - & - & - & 0.9 & 1.2 & - \\
\hline
\end{tabular}

\section{Comparison with theory}

In this section, the experimental results discussed in Section 6 are compared to the theoretical ones obtained in [7]. More precisely, as all the qualitative features predicted by the simple truncated 3-degrees-of-freedom model of Equation (6a)-(6c) have been observed in the experiments, we are now in position for fitting the parameters of this generic model to the experimental resonance curves. This enables, in particular, the estimation of the nonlinear quadratic coefficients $\left\{\alpha_{i}\right\}_{i=1 \ldots, 4}$ that will be compared to the ones obtained in the theoretical case of a perfect shell with perfectly free-edge boundary conditions.

\subsection{Fitting of the theory on the experiments}

Table 4 gathers the parameter values identified from the various experiments. The first column recalls the natural frequencies and the values of coefficients $\left\{\alpha_{i}\right\}_{i=1, \ldots, 4}$ obtained in [7] for a perfect spherical cap (the values of the natural frequencies are those of Table 2). The second column is obtained with a usual modal analysis procedure, performed with the LMS CadaX software and with an acoustic excitation of the structure of low level so that the response of the structure is linear. It enabled the estimation of the natural frequencies and the damping factors of the three involved modes. The five last columns gathers the parameter values identified with Figs. 13-17, by adjusting their values so that the theoretical curves fit the experimental points. This procedure is done by using the remarkable properties of the theoretical frequency response curves enumerated in Section 4.2.

The frequency response curve of Fig. 13 enables the estimation of coefficient $K$ between the current intensity and the force amplitude $F_{\mathrm{dr}}$. A value of $K=$ $0.653 \mathrm{~N} / \mathrm{A}$ was found, by using Equations (15) and the values identified in Section 3 for $\rho, E$, and $\nu$.

\subsection{Results}

First of all, by observing Figs. 13-19, an excellent qualitative agreement is obtained with theory, since all the subtle features of the nonlinear behavior of the system are predicted by the model. In particular, the succession of $\mathrm{SDOF}, \mathrm{C}_{1}$, and $\mathrm{C}_{2}$ solutions during a frequency sweep are well predicted by theory.

However, slight discrepancies on the parameters values are observed, from one experiment to another and a major difference is noticed between the theoretical values of $\left\{\alpha_{i}\right\}_{i=1, \ldots, 4}$ (first column of Table 4 ) and the ones obtained experimentally (other columns). Moreover, the more the forcing level is increased, the less the theoretical curves fit the experimental points. More precisely, one can enumerate the following differences between theory and experiments.

- For large forcing levels ( $F_{\mathrm{dr}}=1.108 \mathrm{~N}$, Fig. 16), the fine geometry of the experimental points is not respected by theory, as well as the limits of the stability frequency bands of the coupled solutions.

- For forcing levels large enough to obtain theoretically an exchange of stability, a small frequency 

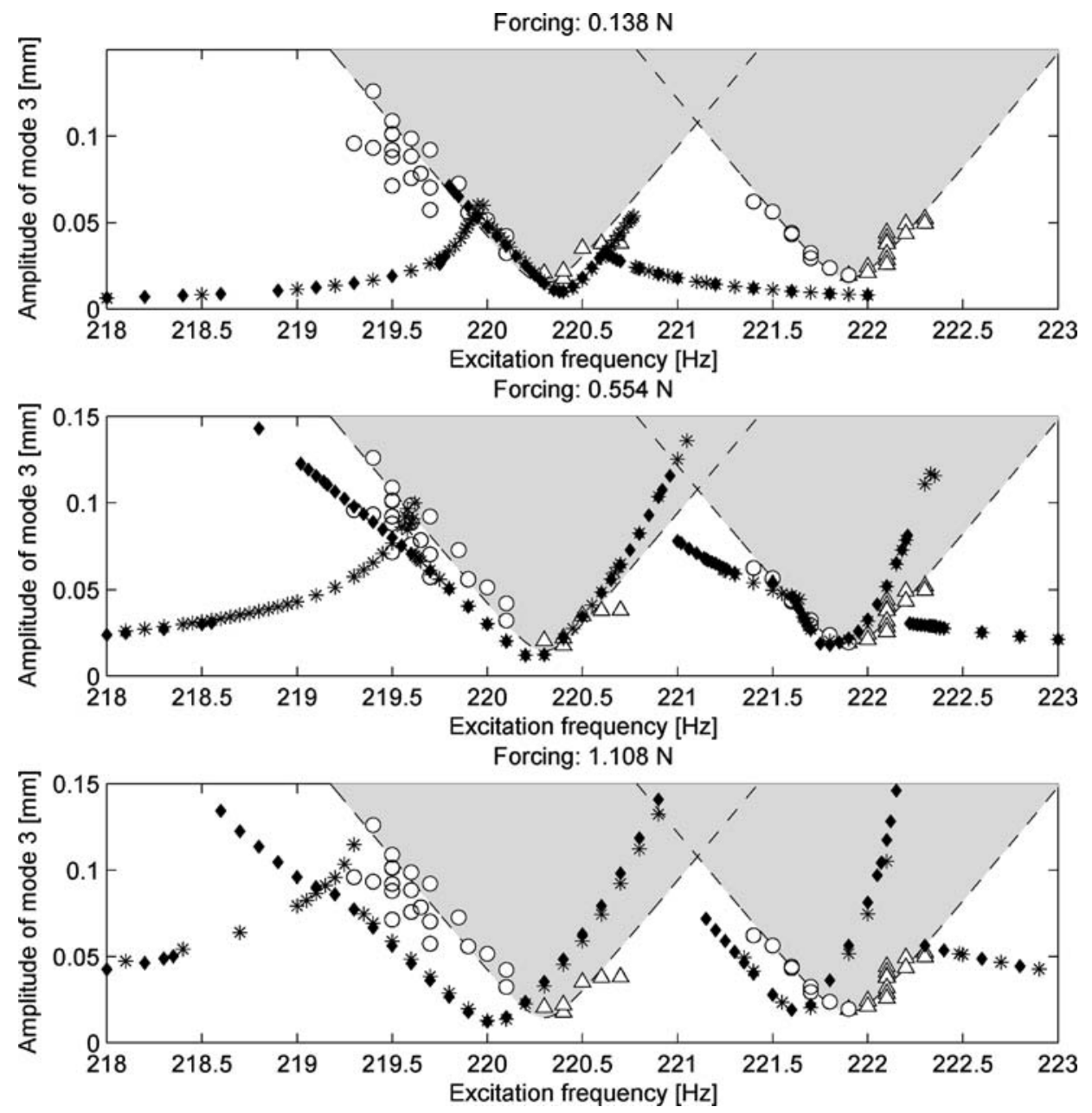

Fig. 20 Superposition of the experimental $a_{3}$ branches of $\mathrm{C}_{1}$ and $\mathrm{C}_{2}$ solutions with the experimental boundary of the instability region, for three forcing levels

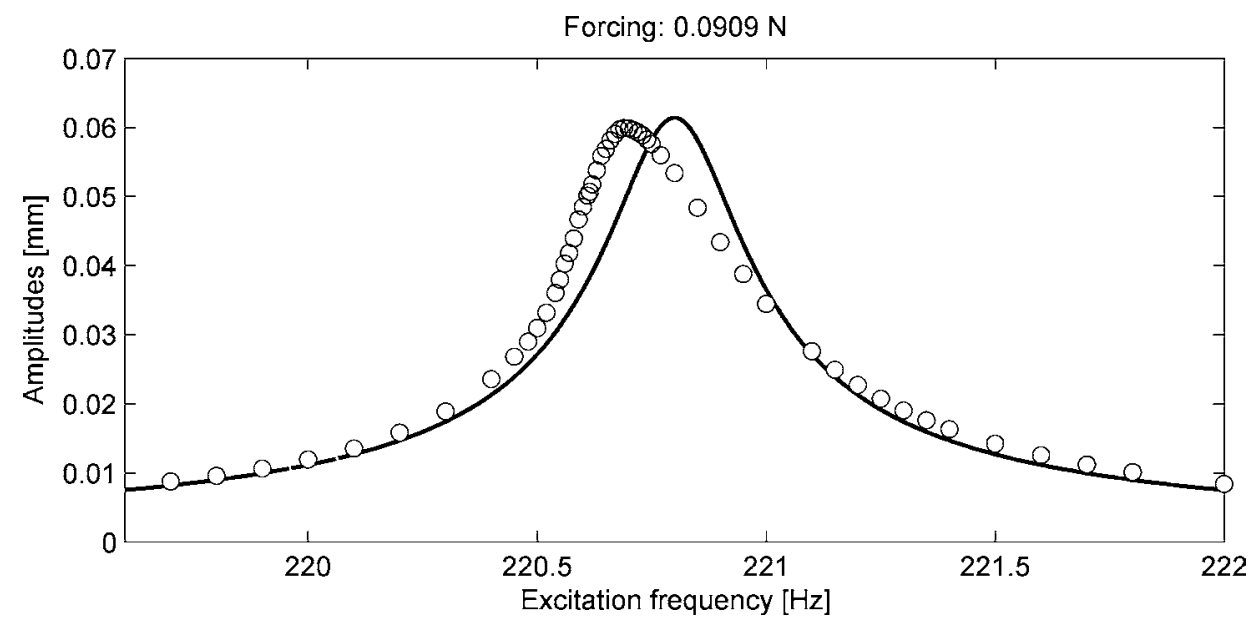

Fig. 21 Frequency response curve for $F_{\mathrm{dr}}=0.0909$ N. (-) $a_{3}$ from theory. (०) $a_{3}$ obtained with an increasing frequency sweep 


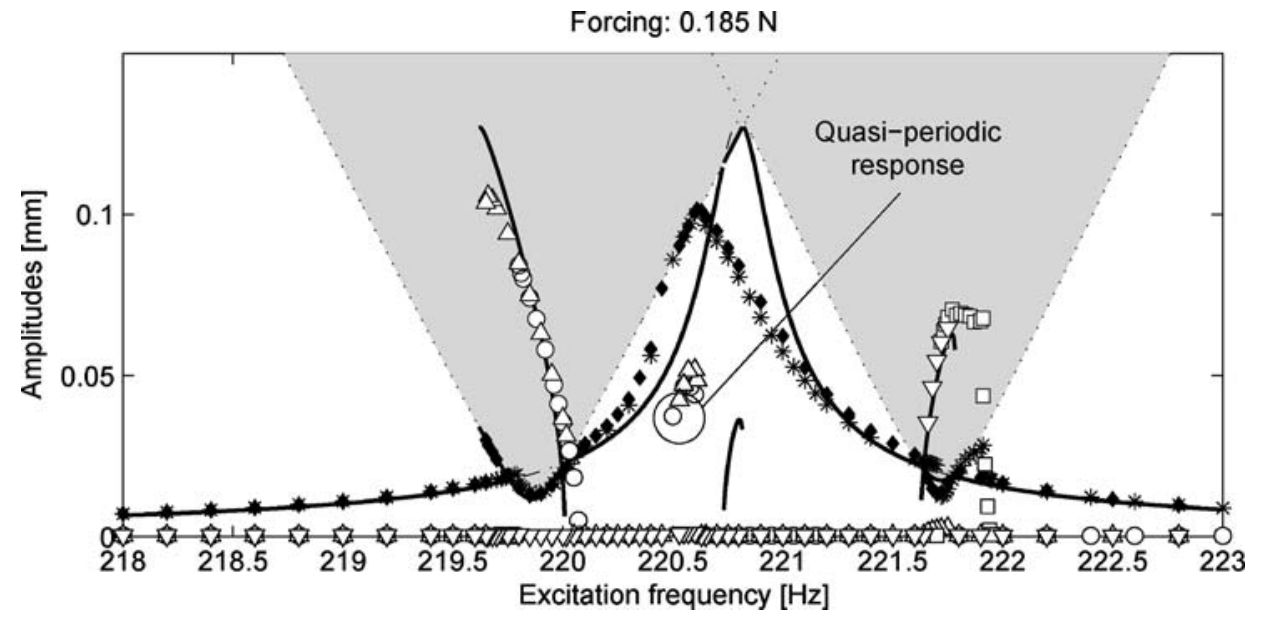

Fig. 22 Frequency response curve, for $F_{\mathrm{dr}}=0.185 \mathrm{~N}$, (-) $a_{1}$, $\gamma_{1}, a_{2}, \gamma_{2}, a_{3}$, and $\gamma_{3}$ from theory, (०) $a_{1}$ and $\gamma_{1},(\square) a_{2}$ and $\gamma_{2}$, (*) $a_{3}$ and $\gamma_{3}$, experiments with an increasing frequency sweep,

$(\triangle) a_{1}$ and $\gamma_{1},(\nabla) a_{2}$ and $\gamma_{2},(\diamond) a_{3}$ and $\gamma_{3}$, experiments with a decreasing frequency sweep

Fig. 23 Time response of accelerometer $A$ in steady state, for the frequency response curve of Fig. 22 $\left(F_{\mathrm{dr}}=0.185 \mathrm{~N}\right)$, in the second $\mathrm{C}_{1}$ solution, with $f_{\text {dr }}=220.5 \mathrm{~Hz}$
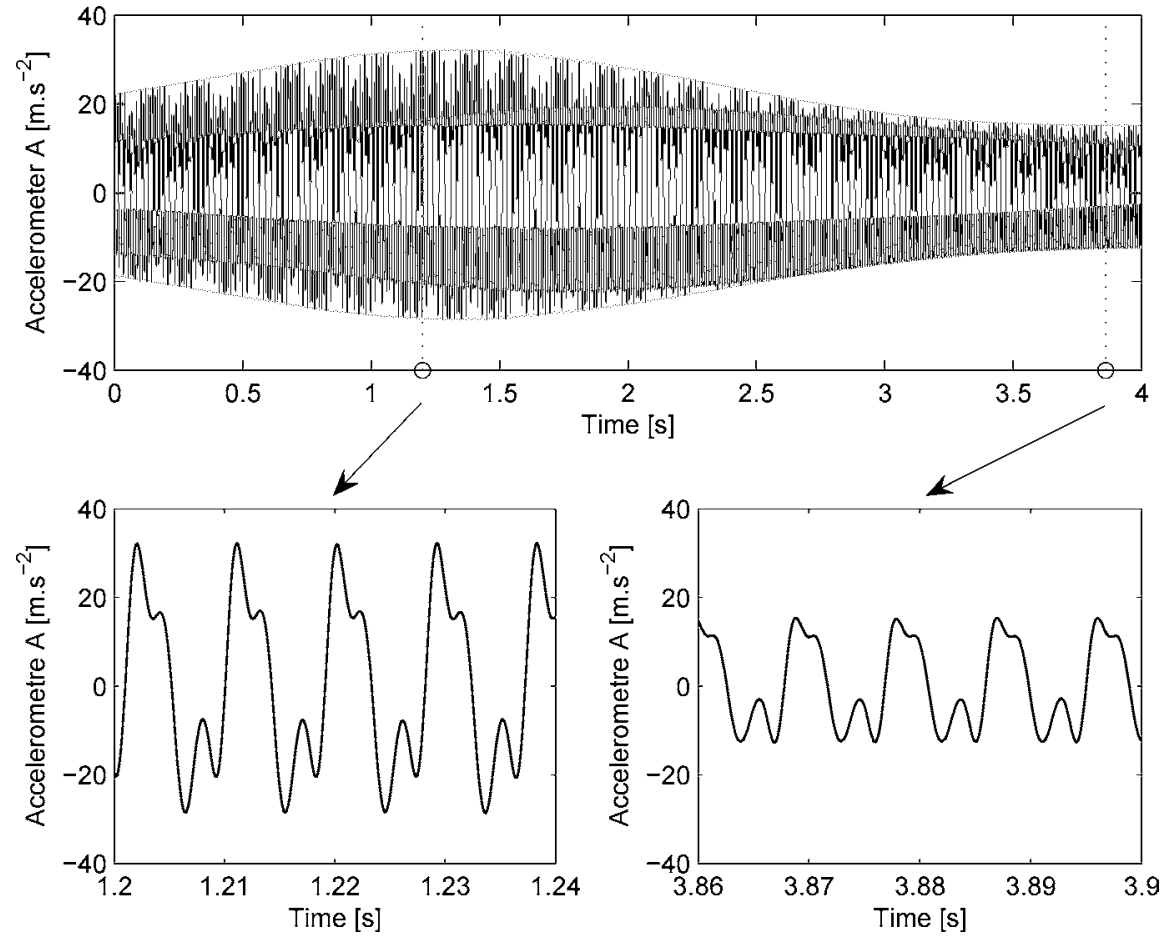

band where both coupled solutions coexist is obtained. This is the case for $F_{\mathrm{dr}}=1.108 \mathrm{~N}$ (Fig. 16) for the interval [221.1, 221.3] Hz. This feature is not in agreement with theory.

- In the experiments, $a_{3}$ branches for $\mathrm{C}_{1}$ and $\mathrm{C}_{2}$ solutions do not coincide with the boundary of the instability region, which is the case for theory. By observing Fig. 20, it seems that $a_{3}$ branches for $\mathrm{C}_{1}$ and $\mathrm{C}_{2}$ solutions are translated toward the low frequencies when the forcing level is increased.

- Figure 21 shows a frequency response curve where only a SDOF solution is obtained, for a forcing level $\left(F_{\mathrm{dr}}=0.091 \mathrm{~N}\right)$ greater than the one of Fig. 13. In this case, one can observe that the SDOF solution is curved toward the low frequencies, as if it was governed by a softening nonlinear behavior. Another 
example is the frequency response curve of Fig. 22, obtained with a forcing level of $F_{\mathrm{dr}}=0.185 \mathrm{~N}$. Those two measurements have been obtained by slightly decreasing the added mass so that the natural frequency of mode 3 is increased, to a value of $220.8 \mathrm{~Hz}$, close to $\hat{f}$, the frequency of the meeting point of $\mathcal{L}_{1}$ and $\mathcal{L}_{2}$. Thus, the SDOF solution can be observed for larger forcing levels than those of the experiments of Figs. 13-16. On can notice that in the case of Fig. 22, due to the particular meeting of the SDOF solution and the instability region, the succession of vibratory regimes is SDOF, $\mathrm{C}_{1}$, SDOF, $\mathrm{C}_{1}, \mathrm{SDOF}$, $\mathrm{C}_{2}$, and SDOF when one follows an increasing frequency sweep. Due to the curvature of the SDOF solution, the theoretical second $\mathrm{C}_{1}$ solution does not fit the corresponding experimental points (around $f_{\mathrm{dr}}=220.6 \mathrm{~Hz}$ ).

In addition, some solutions with amplitude modulations have been noticed for some localized frequency bands. An example is shown in Fig. 23. This amplitude modulation has been observed in a frequency band approximately $0.1 \mathrm{~Hz}$ large centered on $220.5 \mathrm{~Hz}$, at the lowest frequencies of the second $\mathrm{C}_{1}$ solutions of the frequency response curve of Fig. 22.

As a consequence, three main conclusions on the validity of the model can be formulated.

- The qualitative agreement between the theoretical and experimental frequency response curves lead to conclude that even if it is drastic, the 3-degrees-offreedom model described by Equations (5) and (6a)(6c) is a good first approximation of the behavior of the system.

- Due to the curvature of some SDOF solutions, the cubic terms in (6), neglected in the model, should be taken into account, as this feature is a characteristic of a cubic nonlinearity of the softening type $[17,18]$. The correction brought by the cubic terms is of the same order of magnitude as the nonresonant terms that couple the three involved modes to the other modes of the system. For this reason, the formalism of nonlinear modes could be used to keep a 3-degrees-of-freedom model [19]. Using a model including the cubic terms will probably enable to predict most of the previously enumerated features, especially the translation of $a_{3}$ branches as the forcing level increases.

- The imperfections of the curvature (Fig. 2) of the shell have to be taken into account. Even if those imperfections seems to be slight, some major differences with theory have been noticed for all the mode shapes with at least one nodal circle (Fig. 6(e) and (h)) as well as for the values of the corresponding natural frequencies (Table 2). This could explain the major discrepancies between the theoretical values of coefficients $\left\{\alpha_{i}\right\}_{i=1, \ldots, 4}$ with respect to the ones obtained experimentally (Table 4).

\section{Conclusion}

The main goal of this paper was to present an experimental validation of a theoretical model of large amplitude vibration of thin spherical shells exposed in a previous work [7]. The particular vibratory regimes arising from a 1:1:2 internal resonance, between two asymmetric companion modes and one axisymmetric mode, have particularly been addressed. Some resonance curves showing the amplitudes and the phases of the three involved modes, as functions of the driving frequency and with a constant forcing level, have been presented. The vibratory patterns of the shell in the nonlinear coupled regimes have also been measured with a scanning laser vibrometer.

The measurements have been compared to theory and an excellent qualitative agreement was found. In particular, all subtle successions of coupled solutions were obtained experimentally in a manner similar to those predicted by theory. However, some quantitative discrepancies were noticed. Firstly, a major difference has been noticed between the theoretical and experimental values of the coefficients that govern the energy transfers between the three involved modes. Secondly, some discrepancies in the geometry of the coupled branches of solution curves were observed, becoming larger with the excitation force level.

Considering those discrepancies, improvements of the model were proposed. Firstly, it was found that the imperfections of the geometry of the shell, slight in appearance, seem to have a major effect on the vibratory response of the shell. This conclusion was observed in a previous study of the authors when measuring the hardening and softening behavior of the shell [20]. As a consequence, it should be necessary to take into account in the model the real geometry of the shell. This could be done either with a finite-element formulation or by expanding the geometrical imperfections on the modes of a perfect spherical cap. Secondly, it was found 
that the influence of the cubic terms arising from the geometrical nonlinearities seemed to be important and thus should be included in the model, in order to recover the particular features associated to cubic terms. Those terms, that appear in the full version of Equations 6 (see [7]), are of the same order of magnitude as all the nonresonant terms that couple the three modes involved in the internal resonance to the other modes of the structure. As a consequence, the formalism of nonlinear modes (see, e.g., $[19,21])$ could be used to overcomes the errors associated to too sever truncations and to keep a 3-degrees-of-freedom efficient reduced order model. An improved model that takes into account the geometrical imperfections as well as the cubic nonlinear terms is in progress and will soon be submitted.

Acknowledgements Part of this work was performed during a post-doctoral stay of the first author at ENSTA-UME. The authors want to thank Mathieu Fontaine who did the experimental modal analyzes during his master thesis at ENSTA-UME. Antoine Chaigne is also thanked for the loaning of the spherical cap and, above all, for his long time support and his wise advices. The authors are grateful to the reviewers as well as to Julien Ducarne for their interesting comment about the motion of the center of mass of the shell.

\section{Appendix A: Computation of the center of mass of mode shapes}

Deformed shape $\Phi$ of the shell can be represented by a surface of equation $z=\Phi(r, \theta)$, where $(r, \theta, z)$ are the cylindrical coordinates of any point of the surface, with $(r, \theta) \in[0,1] \times\left[0,2 \pi\left[\right.\right.$. The vertical coordinate $z_{m}$ of the center of mass of mode shape $\Phi$ is defined by:

$m z_{m}=\int_{\mathcal{S}} z d m$,

where $\mathcal{S}$ is the surface of the mode shape, $m$ the total mass, and $d m$ is a mass surface element. $m$ and $d m$ have to be defined on the undeformed configuration of the structure, so that $m=\rho h \pi a^{2}$ and $d m=\rho h r d \theta d r$. If $\Phi$ is axisymmetric, it writes $\Phi(r, \theta)=\Phi(r)$ and one obtains:

$z_{m}=\frac{2}{a^{2}} \int_{0}^{a} r \Phi(r) d r$.
Equation (17) has been applied to the experimental profiles of the mode shapes shown in Fig. 6(e), and the integrals have been numerically computed by the trapezoidal method.

\section{Appendix B: Theoretical amplitudes and phases}

This section briefly recalls the theoretical expressions of $\left\{\bar{a}_{i}\right\}_{i=1,2,3}$ exposed in [7] and adds the ones for $\left\{\gamma_{i}\right\}_{i=1,2,3}$. The following detuning parameters are introduced:

$$
\begin{aligned}
\bar{\omega}_{2} & =\bar{\omega}_{1}+\varepsilon \sigma_{0}, \quad \bar{\omega}_{3}=2 \bar{\omega}_{1}+\varepsilon \sigma_{1}, \\
\bar{\omega} & =\bar{\omega}_{3}+\varepsilon \sigma_{2} .
\end{aligned}
$$

\section{B.1 SDOF solution}

For the SDOF solution, $\bar{a}_{1} \equiv \bar{a}_{2} \equiv 0$. One obtains:

$\bar{a}_{3}=\frac{Q}{2 \bar{\omega}_{3} \sqrt{\sigma_{2}^{2}+\xi_{3}^{2} \bar{\omega}_{3}^{2}}}, \quad \gamma_{3}=-\arctan \frac{\xi_{3} \bar{\omega}_{3}}{\sigma_{2}}$.

\section{B.2 $\mathrm{C}_{1}$ solution}

For $\mathrm{C}_{1}$ solution, $\bar{a}_{2} \equiv 0$. One obtains:

$\bar{a}_{3}=\frac{2 \bar{\omega}_{1}}{\alpha_{1}} \sqrt{4 \xi_{1}^{2} \bar{\omega}_{1}^{2}+\left(\sigma_{1}+\sigma_{2}\right)^{2}}$,
$\gamma_{3}=\arctan \frac{2\left(\alpha_{3} \xi_{1} \bar{\omega}_{1}^{2} \bar{a}_{1}^{2}+\alpha_{1} \xi_{3} \bar{\omega}_{3}^{2} \bar{a}_{3}^{2}\right)}{\left(\sigma_{1}+\sigma_{2}\right) \alpha_{3} \bar{\omega}_{1} \bar{a}_{1}^{2}-2 \sigma_{2} \alpha_{1} \bar{\omega}_{3} \bar{a}_{3}^{2}}$,

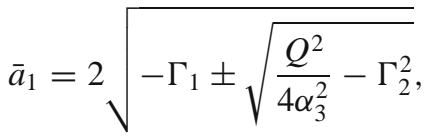

$\gamma_{1}=-\arctan \frac{2 \xi_{1} \bar{\omega}_{1}}{\sigma_{1}+\sigma_{2}}$,

with

$\Gamma_{1}=\frac{2 \bar{\omega}_{1} \bar{\omega}_{3}}{\alpha_{1} \alpha_{3}}\left[2 \mu_{1} \mu_{3}-\sigma_{2}\left(\sigma_{1}+\sigma_{2}\right)\right]$,

$\Gamma_{2}=\frac{2 \bar{\omega}_{1} \bar{\omega}_{3}}{\alpha_{1} \alpha_{3}}\left[2 \sigma_{2} \mu_{1}+\mu_{3}\left(\sigma_{1}+\sigma_{2}\right)\right]$. 


\section{B. $3 \mathrm{C}_{2}$ solution}

For $\mathrm{C}_{2}$ solution, $\bar{a}_{1} \equiv 0$. One obtains:

$\bar{a}_{3}=\frac{2 \bar{\omega}_{2}}{\alpha_{2}} \sqrt{4 \xi_{2}^{2} \bar{\omega}_{2}^{2}+\left(\sigma_{1}+\sigma_{2}-2 \sigma_{0}\right)^{2}}$,

$\gamma_{3}=\arctan \frac{2\left(\alpha_{4} \xi_{2} \bar{\omega}_{2}^{2} \bar{a}_{2}^{2}+\alpha_{2} \xi_{3} \bar{\omega}_{3}^{2} a_{3}^{2}\right)}{\left(\sigma_{1}+\sigma_{2}-2 \sigma_{0}\right) \alpha_{4} \bar{\omega}_{2} \bar{a}_{2}^{2}-2 \sigma_{2} \alpha_{2} \bar{\omega}_{3} \bar{a}_{3}^{2}}$,

$\bar{a}_{2}=2 \sqrt{-\Gamma_{3} \pm \sqrt{\frac{Q^{2}}{4 \alpha_{4}^{2}}-\Gamma_{4}^{2}}}$,

$\gamma_{2}=-\arctan \frac{2 \xi_{2} \bar{\omega}_{2}}{\sigma_{1}+\sigma_{2}-2 \sigma_{0}}$,

with

$$
\begin{aligned}
\Gamma_{3} & =\frac{2 \bar{\omega}_{2} \bar{\omega}_{3}}{\alpha_{2} \alpha_{4}}\left[2 \mu_{2} \mu_{3}-\sigma_{2}\left(\sigma_{1}+\sigma_{2}-2 \sigma_{0}\right)\right], \\
\Gamma_{4} & =\frac{2 \bar{\omega}_{2} \bar{\omega}_{3}}{\alpha_{2} \alpha_{4}}\left[2 \sigma_{2} \mu_{2}+\mu_{3}\left(\sigma_{1}+\sigma_{2}-2 \sigma_{0}\right)\right] .
\end{aligned}
$$

\section{Appendix C: Measurement of the phases differences}

All three transducer signals are processed by identical filters, whose common cutoff frequency has been adjusted to $220 \mathrm{~Hz}$, in order to select the fundamental component of the accelerometer signals (of frequency $f_{\mathrm{dr}} / 2$ ) as well as the fundamental component of the vibrometer signal (of frequency $f_{\mathrm{dr}}$ ). After filtering, the time evolution of $s_{A}$ and $s_{C}$ (defined by Equations (12)) have the shapes shown on Fig. 24. Times $t_{A}$ and $t_{C}$

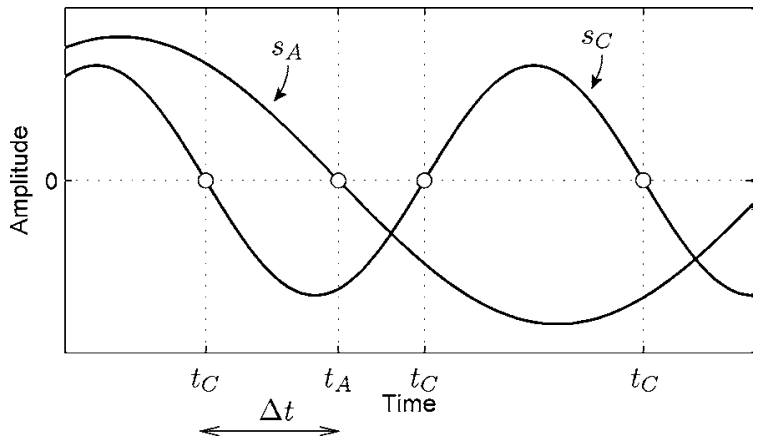

Fig. 24 Time evolution of $s_{A}$ and $s_{C}$ where those signals are zero fulfill the following relationships:

$2 \pi f_{\mathrm{dr}} t_{A}=2 \varphi_{A}+\pi+2 \lambda_{A} \pi$,

$2 \pi f_{\mathrm{dr}} t_{C}=\varphi_{C}+\frac{\pi}{2}+\lambda_{C} \pi$,

where $\lambda_{A}$ and $\lambda_{C}$ are two integers. By considering that $s_{A}$ is an acceleration and $s_{C}$ is a velocity, one obtains

$$
\begin{aligned}
\frac{\gamma_{1}+\gamma_{3}}{2} & =\varphi_{A}-\varphi\left(f_{\mathrm{dr}} / 2\right), \\
\gamma_{3} & =\varphi_{C}-\varphi\left(f_{\mathrm{dr}}\right),
\end{aligned}
$$

where $\varphi(f)$ is the phase difference between the output and the input of the filter used to process the accelerometer and vibrometer signals, whose response function is shown on Fig. 25. As a consequence, by using Equations (32) and (33), one obtains:

$\gamma_{1}=2 \pi f_{\mathrm{dr}} \Delta t-2 \varphi\left(f_{\mathrm{dr}} / 2\right)+\varphi\left(f_{\mathrm{dr}}\right)+\lambda \pi$,

where $\Delta_{t}=t_{A}-t_{C}$ and $\lambda$ is an integer. A measure of $\gamma_{1}$ (and thus $\gamma_{2}$ ) is then obtained by measuring $\Delta t$ with an oscilloscope and corrected it with the phase response of the filter by Equation (34). The value of $\lambda$ is adjusted so that $\gamma_{1}$ and $\left(\gamma_{2}\right)$ falls between 0 and $\pi$.
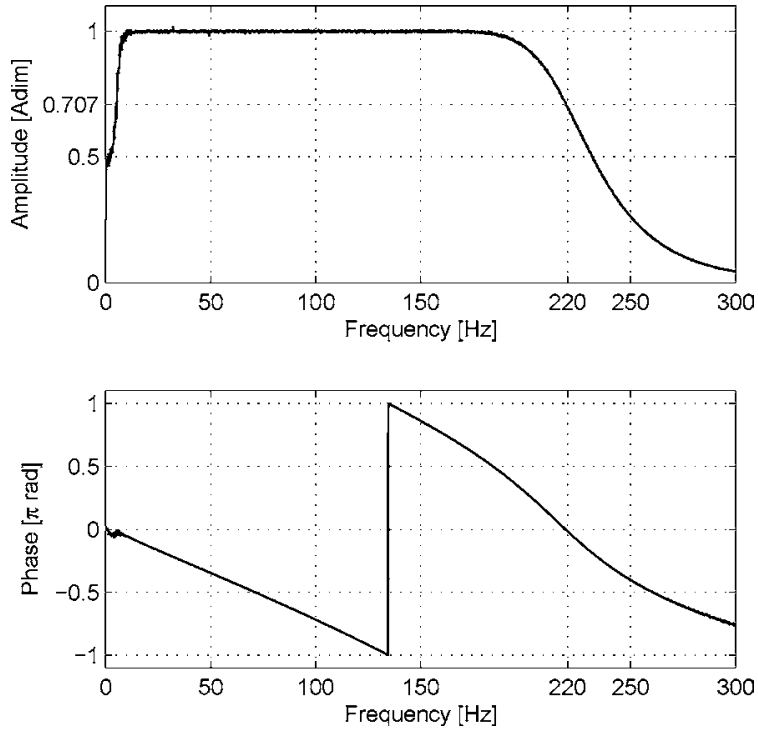

Fig. 25 Input/output frequency response function of the lowpass filter with $220 \mathrm{~Hz}$ cutoff frequency 


\section{Appendix D: Notations and acronyms}

\begin{tabular}{|c|c|}
\hline FRF & Frequency response function \\
\hline PSD & Power spectral density \\
\hline RMS & Root mean square \\
\hline SDOF & $\begin{array}{l}\text { Single-degree-of-freedom vibratory } \\
\text { regime }\end{array}$ \\
\hline $\mathrm{C}_{1}, \mathrm{C}_{2}$ & $\begin{array}{l}\text { Coupled regime respectively with mode } 1 \\
\text { and mode } 2\end{array}$ \\
\hline $\mathcal{L}_{1}, \mathcal{L}_{2}$ & $\begin{array}{l}\text { Boundaries of the instability regions } \\
\text { associated to } C_{1} \text { and } C_{2}\end{array}$ \\
\hline$a, h, H, R$ & $\begin{array}{l}\text { Radius, thickness, center height and } \\
\text { radius of curvature of the shell }\end{array}$ \\
\hline$\rho, E, v$ & $\begin{array}{l}\text { Density, Young's modulus and Poisson's } \\
\text { ration of the shell material }\end{array}$ \\
\hline$K$ & $\begin{array}{l}\text { Proportionality coefficient between the } \\
\text { current intensity in the coil and the } \\
\text { force created on the magnet }\end{array}$ \\
\hline$(k, n)$ & $\begin{array}{l}\text { number of nodal diameters and nodal } \\
\text { circles of a mode shape }\end{array}$ \\
\hline$f_{k n}, \bar{\omega}_{k n}$ & $\begin{array}{l}\text { Dimensioned and dimensionless natural } \\
\text { frequency of mode }(k, n)\end{array}$ \\
\hline$f^{*}$ & $\begin{array}{l}\text { Coefficient between dimensioned and } \\
\text { dimensionless frequencies }\end{array}$ \\
\hline$f_{i}, \bar{\omega}_{i}$ & $\begin{array}{l}\text { Dimensioned and dimensionless natural } \\
\text { frequencies of mode } i\end{array}$ \\
\hline$\mu_{i}, \xi_{i}$ & $\begin{array}{l}\text { Damping coefficient and damping factor } \\
\text { of mode } i\end{array}$ \\
\hline$(r, \theta),(\bar{r}, \theta)$ & $\begin{array}{l}\text { Dimensioned and dimensionless polar } \\
\text { coordinates }\end{array}$ \\
\hline $\bar{w}(\bar{r}, \theta, \bar{t})$ & Dimensionless displacement of the shell \\
\hline$\Phi_{i}(r, \theta)$ & Deflection shape of mode $i$ \\
\hline$R_{k n}(\bar{r})$ & Radial part of the shape of mode $(k, n)$ \\
\hline$q_{i}(t)$ & Modal coordinate of mode $i$ \\
\hline$\alpha_{i}$ & Coefficients of quadratic nonlinear terms \\
\hline$Q$ & Amplitude of forcing term of mode 3 \\
\hline$F_{\mathrm{dr}}$ & Amplitude of driving force \\
\hline$f_{\mathrm{dr}}, \bar{\Omega}$ & $\begin{array}{l}\text { Dimensioned and dimensionless driving } \\
\text { frequencies }\end{array}$ \\
\hline$a_{i}, \bar{a}_{i}$ & $\begin{array}{l}\text { Dimensioned and dimensionless } \\
\text { amplitudes of vibrations of mode } i\end{array}$ \\
\hline$\gamma_{i}$ & Phase of mode $i$ \\
\hline$\hat{f}, \hat{\omega}$ & $\begin{array}{l}\text { Dimensioned and dimensionless } \\
\text { frequency of meeting of the instability } \\
\text { region boundaries }\end{array}$ \\
\hline$w_{\alpha}(t), \bar{w}_{\alpha}(\bar{t})$ & $\begin{array}{l}\text { Dimensioned and dimensionless } \\
\text { displacement of transducer } \alpha\end{array}$ \\
\hline$\left(\bar{r}_{m}, \theta_{A}\right),\left(\bar{r}_{m}, \theta_{B}\right)$ & Location of accelerometers $A$ and $B$ \\
\hline$s_{\alpha}(t)$ & Signal measured by transducer $\alpha$ \\
\hline$\left(s_{\alpha}^{0}, \varphi_{\alpha}\right)$ & RMS amplitude and phase of $s_{\alpha}$ \\
\hline$I$ & $\begin{array}{l}\text { RMS amplitude of the current intensity in } \\
\text { the coil }\end{array}$ \\
\hline
\end{tabular}

\section{References}

1. Nayfeh, A.H.: Nonlinear Interactions. Wiley, New York (2000)

2. Touzé, C., Chaigne, A.: Lyapunov exponents from experimental time series: Application to cymbal vibrations. Acustica 86, 557-567 (2000)

3. Chaigne, A., Touzé, C., Thomas, O.: Nonlinear vibrations and chaos in gongs and cymbals. Acoust. Sci. Technol. 26(5), 403-409 (2005)

4. Qatu, M.S.: Recent research advances in the dynamic behavior of shells: 1989-2000: Part 2. Homogeneous shells. ASME Appl. Mech. Rev. 55(5), 415-434 (2002)

5. Amabili, M., Païdoussis, M.P.: Review of studies on geometrically nonlinear vibrations and dynamics of circular cylindrical shells and panels, with and without fluid-structure interaction. ASME Appl. Mech. Rev. 56(4), 349-381 (2003)

6. Alhazza, K.A., Alhazza, A.A.: A review of the vibrations of plates and shells. Shock Vib. Dig. 36(5), 377-395 (2004)

7. Thomas, O., Touzé, C., Chaigne, A.: Non-linear vibrations of free-edge thin spherical shells: Modal interaction rules and 1:1:2 internal resonance. Int. J. Sol. Struct. 42(11-12), 3339-3373 (2005)

8. Hoppmann, W.H., II, Baronet, C.N.: A study of the vibrations of shallow spherical shells. J. Appl. Mech. 30(3), 329-334 (1963)

9. Amabili, M., Pellegrini, M., Tommesani, M.: Experiments on largeamplitude vibrations of a circular cylindrical panel. J. Sound Vib. 260(3), 537-547 (2003)

10. Amabili, M.: Theory and experiments for large-amplitude vibrations of empty and fluid-filled circular cylindrical shells with imperfections. J. Sound Vib. 262(4), 921-975 (2003)

11. Evensen, H.A., Evan-Iwanowsky, R.M.: Dynamic response and stability of shallow spherical shells subject to timedependant loading. AIAA J. 5(5), 969-976 (1967)

12. Yasuda, K., Kushida, G.: Nonlinear forced oscillations of a shallow spherical shell. Bull. JSME 27(232), 2233-2240 (1984)

13. Touzé, C., Thomas, O., Chaigne, A.: Asymmetric non-linear forced vibrations of free-edge circular plates. Part 1: Theory. J. Sound Vib. 258(4), 649-676 (2002)

14. Thomas, O., Touzé, C., Chaigne, A.: Asymmetric non-linear forced vibrations of free-edge circular plates. Part 2: Experiments. J. Sound Vib. 265(5), 1075-1101 (2003)

15. Chu, H.-N., Herrmann, G.: Influence of large amplitudes on free flexural vibrations of rectangular elastic plates. J. Appl. Mech. 23, 532-540 (1956)

16. Efstathiades, G.J.: A new approach to the large-deflection vibrations of imperfect circular disks using Galerkin's procedure. J. Sound Vib. 16(2), 231-253 (1971)

17. Nayfeh, A.H., Mook, D.T.: Nonlinear Oscillations. Wiley, New York (1979)

18. Touzé, C., Thomas, O.: Non-linear behaviour of free-edge shallow spherical shells: Effect of the geometry. Int. J. NonLinear Mech. 41(5), 678-692 (2006)

19. Touzé, C., Thomas, O., Chaigne, A.: Hardening/softening behaviour in non-linear oscillations of structural systems using non-linear normal modes. J. Sound Vib. 273(1-2), 77$101(2004)$ 
20. Touzé, C., Thomas, O.: Type of non-linearity of shallow spherical shells using non-linear normal modes. In: Fifth Euromech Non-linear Dynamics Conference (ENOC 2005), Eindhoven, August (2005)
21. Touzé, C., Thomas, O., Huberdeau, A.: Asymptotic nonlinear normal modes for large amplitude vibrations of continuous structures. Comput. Struct. 82(31-32), 2671-2682 (2004) 